\title{
Study of partial and average conditional Kendall's tau
}

https://doi.org/10.1515/demo-2021-0104

Received June 22, 2020; accepted May 17, 2021

Abstract: When the interest is in studying conditional dependencies, and more precisely the strength of conditional dependencies, some kind of averaging over the conditioning random vector may be needed. Examples of average measures that can serve in this context are the average conditional Kendall's tau and partial Kendall's tau. It is known that these measures differ in general. Some statistical tests are based on these average measures, and a better knowledge of them is of importance. The aim of this paper is to provide a quantitative study of the possible differences of these two average measures, and to establish sufficient conditions under which they coincide. Both measures are studied in two fairly general settings. In each setting theoretical results are established as well as several illustrative examples given.

Keywords: average conditional copula, association measures, conditional copula, Kendall's tau, partial copula

MSC: Primary 60Exx, Secondary 62Hxx

\section{Introduction}

Dependencies between random variables are fully characterized by the copula function. Standard references for theoretical background on and applications of copulas include the books [5], [28], [9], and for copula modeling using the R software [23]. Similarly conditional dependencies between variables given the realized value of a covariate $\boldsymbol{X}=\left(X_{1}, \ldots, X_{d}\right)^{\mathrm{T}}$ is completely captured by the conditional copula, denoted by $C_{\boldsymbol{X}}$ in this paper. See [29] for an early reference on the concept of conditional copulas.

While copulas give a full description of a dependence structure of two or more random variables, association measures are tools to measure how strong the association is between them. Most well-known association measures can be expressed in terms of the copula function. See, for example, [28]. The same holds for their corresponding conditional versions. See, for example, [35] and [21] for a brief overview of conditional association measures, and approaches to estimate these nonparametrically. For conditional dependencies an association measure varies with the value of the covariate $\boldsymbol{X}=\left(X_{1}, \ldots, X_{d}\right)^{\mathrm{T}}$, and comparing different conditional associations, conditionally upon the same covariate $\boldsymbol{X}$, would thus result into comparing functions (in $\boldsymbol{X}$ ).

When the interest is in the strength of the conditional dependence a possible approach is to look into some kind of average of the conditional dependence. This led to the concept of average conditional copula, that was first mentioned by [2] and [3], and further studied in [19] and [32], among others. Suppose that the interest is in the dependence between the random variables $Y_{1}$ and $Y_{2}$ conditionally upon a realized value of $\boldsymbol{X}$. Then, if $Y_{1}$ and $Y_{2}$ have continuous marginal cumulative distribution functions, the average conditional

*Corresponding Author: Irène Gijbels: Department of Mathematics and Leuven Statistics Research Center (LStat), KU Leuven, Celestijnenlaan 200B, Box 2400, B-3001 Leuven (Heverlee), Belgium, E-mail: irene.gijbels@kuleuven.be Margot Matterne: Department of Mathematics and Leuven Statistics Research Center (LStat), KU Leuven, Celestijnenlaan 200B, Box 2400, B-3001 Leuven (Heverlee), Belgium 
copula of $\left(Y_{1}, Y_{2}\right)$ given $\boldsymbol{X}$ defined as $\mathrm{E}_{\boldsymbol{X}}\left[C_{\boldsymbol{X}}\right]$ coincides with a partial copula. The latter quantity is defined as the copula of $F_{1 X}\left(Y_{1}\right)$ and $F_{2 X}\left(Y_{2}\right)$, where $F_{j X}$, for $j=1,2$, denotes the conditional cumulative distribution function of $Y_{j}$ given $\boldsymbol{X}$. Average conditional copulas and partial copulas offer each a way to look into a kind of average of conditional dependency.

When the main interest is in comparing strengths of conditional dependencies, there are at least two ways to proceed: (i) considering the average of the conditional association measure; or (ii) considering the association measure based on the partial copula. Both ways lead to measuring an average type of strength in conditional dependencies, and are respectively referred to in the literature as average conditional association measures and partial association measures. See [19] for background information. As was argued in that paper, among others, for several association measures both concepts of average measures coincide. This however is not the case for Kendall's tau, which is among the oldest and well-studied association measures. Whether, for a given association measure, the average conditional association measure equals the partial association measure, or not, is related to the fact whether an association measure is a linear functional of the copula or not. Spearman's rho and Blomqvist's beta are linear functionals of the copula, whereas Kendall's tau is not. See [10], [11] and [16], for association measures in case of bivariate measures of concordance. See also Section 8 for some further discussions.

In the copula literature a popular assumption often made (in particular in applications), is that the conditional dependence structure between $Y_{1}$ and $Y_{2}$ is not varying with the covariate $\boldsymbol{X}$. The only possible influence of $\boldsymbol{X}$ is on the marginal distributions of $Y_{1}$ and $Y_{2}$. This assumption, referred to as the simplifying assumption, is very convenient since it simplifies things a lot. However, such a serious simplification can have an impact on the analysis and conclusions, and needs to be verified or justified. See [1], [8] and [27], among others, for recent contributions to evaluations and criticism regarding the simplifying assumption. As will be argued in Section 2, the average conditional Kendall's tau and the partial Kendall's tau coincide in case the simplifying assumption holds. Therefore the difference between estimates of the two concepts can be used as a basis to build test statistics to test the null hypothesis that the simplifying assumption holds. This is a first reason for studying the concepts of average conditional Kendall's tau and partial Kendall's tau. A second reason is the general interest in measures of associations that summarize the strength of conditional dependencies, taking into account influences of covariates. Since the average conditional Kendall's tau and the partial Kendall's tau constitute two such measures of association, knowledge about when and how they differ is important.

As an illustrative example, consider random variables $Y_{1}$ and $Y_{2}$ that are both influenced by a (onedimensional) covariate $X$ (with support [0,1]), via regression models $Y_{1}=m(X)+\varepsilon_{1}$, and $Y_{2}=m(X)+$ $\varepsilon_{2}$, with $m(X)$ some regression function, and where the error terms $\varepsilon_{1}$ and $\varepsilon_{2}$ are uniformly distributed on $[0,1]$. The joint distribution function of the error terms, conditionally upon $X=x$ is given by a mixture of the independence copula $\left(\Pi\left(u_{1}, u_{2}\right)=u_{1} u_{2}\right)$ and the comonotonic copula $\left(M\left(u_{1}, u_{2}\right)=\min \left(u_{1}, u_{2}\right)\right)$, with mixing proportions $x$ and $1-x$ respectively. In other words, the conditional joint distribution function of $\left(\varepsilon_{1}, \varepsilon_{2}\right)$ given $X=x$ is then given by $x \Pi\left(u_{1}, u_{2}\right)+(1-x) M\left(u_{1}, u_{2}\right)$. For values of $x$ close to 1 , we are closer to a conditional independence between $Y_{1}$ and $Y_{2}$ (i.e. independent error terms), whereas for values of $x$ close to zero, the conditional dependence structure between $Y_{1}$ and $Y_{2}$ approaches that of a perfect linear relationship. See also Figure 2 in Section 2.3. Suppose now that $X$ has a Beta distribution with parameters $(a, b)$. Then it can be shown that the average conditional Kendall's tau equals $[(3+2 a+3 b) b] /[3(a+b)(a+b+1)]$ whereas the partial Kendall's tau equals $[(2 a+3 b) b] /\left[3(a+b)^{2}\right]$. In this example the difference between the average conditional Kendall's tau and partial Kendall's tau is $[a b] /\left[3(a+b)^{2}(a+b+1)\right]$. When $X$ has a uniform distribution (i.e. $a=b=1$ ) this leads to a difference of $1 /(36)$ between the two measures; and for any symmetric Beta distribution (i.e. $a=b)$ this gives a difference of $1 /[12(2 a+1)]$, for example. From one of the theoretical results established in this paper, it appears that an upperbound for the difference in this setting is $1 /(12)$.

The aim of this paper is to study in detail the average conditional Kendall's tau and the partial Kendall's tau. The main contributions in this paper consist of: (i) providing settings and sufficient conditions under which both concepts coincide; (ii) providing insights in what drives the difference between the two average 
measures; (iii) providing provided bounds on their possible differences. For simplicity of presentation we focus mostly on bivariate copulas, but also discuss the extension of (part of) the results to multivariate copulas.

The paper is organized as follows. In Section 2 we briefly describe conditional and partial copulas and association measures. In this section we also introduce the framework of two settings on which we focus in this work: a first general setting of a mixture of copulas, and a second setting of an overarching Archimedean copula structure. In Sections 3 and 4 we then study in detail, in respectively these two settings, the average conditional Kendall's tau and the partial Kendall's tau. Some more insights on bounds of their differences are provided in Section 5. A theoretical investigation of the orderings between partial and average conditional Kendall's tau is given in Section 6. The extension of the results to multivariate copulas is discussed in Section 7. Proofs of all theoretical results are deferred to the Appendix. Some additional examples, details of some calculations and some further theoretical results are given in the Supplementary Material.

\section{Partial and average conditional Kendall's tau}

\subsection{Conditional copulas and association measures}

Consider two random variables $Y_{1}$ and $Y_{2}$ and a covariate vector $\boldsymbol{X}=\left(X_{1}, \ldots, X_{d}\right)^{\mathrm{T}}$, of dimension $d$, with domain $\mathcal{R}_{\boldsymbol{X}} \subseteq \mathbb{R}^{d}$. Herein $\boldsymbol{A}^{\mathrm{T}}$ denotes the transpose of a vector or matrix $\boldsymbol{A}$. For $\boldsymbol{X}=\left(x_{1}, \ldots, x_{d}\right)^{\mathrm{T}} \in \mathbb{R}^{d}$, the conditional joint distribution function of $\left(Y_{1}, Y_{2}\right)$ given $\boldsymbol{X}=\boldsymbol{x}$ is denoted by $H_{\boldsymbol{x}}$. The conditional marginal distribution functions of $Y_{1}$ and $Y_{2}$ given $\boldsymbol{X}=\boldsymbol{X}$ are denoted by $F_{1 \boldsymbol{x}}$ and $F_{2 \boldsymbol{x}}$ respectively. More precisely

$$
\begin{aligned}
& H_{\boldsymbol{x}}\left(y_{1}, y_{2}\right)=P\left\{Y_{1} \leq y_{1}, Y_{2} \leq y_{2} \mid \boldsymbol{X}=\boldsymbol{x}\right\} \\
& F_{1 \boldsymbol{x}}\left(y_{1}\right)=P\left\{Y_{1} \leq y_{1} \mid \boldsymbol{X}=\boldsymbol{x}\right\}=H_{\boldsymbol{x}}\left(y_{1},+\infty\right) \\
& F_{2 \boldsymbol{x}}\left(y_{2}\right)=P\left\{Y_{2} \leq y_{2} \mid \boldsymbol{X}=\boldsymbol{x}\right\}=H_{\boldsymbol{x}}\left(+\infty, y_{2}\right) .
\end{aligned}
$$

Throughout the paper we assume that the conditional distribution functions $F_{1 x}(y)$ and $F_{2 x}(y)$ are continuous in $y$. For $\boldsymbol{x} \in \mathcal{R}_{\boldsymbol{X}}$ Sklar's theorem [31] applied to conditional probabilities ensures the existence of a unique function $C_{X}:[0,1]^{2} \rightarrow[0,1]$, such that

$$
H_{\boldsymbol{x}}\left(y_{1}, y_{2}\right)=C_{\boldsymbol{x}}\left(F_{1 x}\left(y_{1}\right), F_{2 x}\left(y_{2}\right)\right) \text {. }
$$

The function $C_{\boldsymbol{X}}$ is the conditional copula of $\left(Y_{1}, Y_{2}\right)$ given $\boldsymbol{X}=\boldsymbol{x}$. See [29], [21] and [35], among others. This conditional copula function fully describes the conditional dependence of $Y_{1}$ and $Y_{2}$ given the realized vector value $\boldsymbol{x}$ of the covariate vector $\boldsymbol{X}$. For each realization of $\boldsymbol{X}$ this function exists, and hence for any $\boldsymbol{u}=\left(u_{1}, u_{2}\right)^{\mathrm{T}} \in[0,1]^{2}$, the quantity $C_{\boldsymbol{X}}\left(u_{1}, u_{2}\right)$ can be looked upon as a random vector.

Consider now the setting where the interest is in comparing the conditional dependence in random couples $\left(Y_{1}, Y_{2}\right)$ and $\left(W_{1}, W_{2}\right)$ and this for each couple conditionally upon the event $\boldsymbol{X}=\boldsymbol{x}$. One approach towards evaluations and also comparisons of conditional dependencies is to focus on the strenghts of the conditional dependencies between the components in $\left(Y_{1}, Y_{2}\right)$ and $\left(W_{1}, W_{2}\right)$. A common tool for measuring such strengths is by looking at (conditional) association measures. Among the well-known unconditional association measures are Spearman's rho, Kendall's tau, and Blomqvist's beta to name just a few. See [28, Chapter 5], among others. For all these association measures conditional versions can be given. See [19, Table 1] for an overview of various unconditional and conditional association measures. It is important to note that many conditional association measures can be expressed as a specific functional of the conditional copula. For example, the conditional Spearman's rho and the conditional Kendall's tau can be written respectively as:

$$
\begin{aligned}
& \rho(\boldsymbol{x})=12 \iint C_{\boldsymbol{x}}\left(u_{1}, u_{2}\right) d \boldsymbol{u}-3 \\
& \tau(\boldsymbol{x})=4 \iint C_{\boldsymbol{x}}\left(u_{1}, u_{2}\right) d C_{\boldsymbol{x}}\left(u_{1}, u_{2}\right)-1 .
\end{aligned}
$$

As an illustration we consider a data example concerning a comparison of aspects of photosynthesis in 2746 trees and 1196 lianas in two Panamanian lowland tropical forests. The illustration concerns the 
dependence between temperature (measured on the leaves) in degrees Celcius and the photosynthetic rate expressed in $\mu \mathrm{mol} \mathrm{CO}_{2} \mathrm{~m}^{-2} \mathrm{~s}^{-1}$ ( $\mathrm{m}=$ meter and $\mathrm{s}=$ second). One can think of the couples $\left(Y_{1}, Y_{2}\right)$ and $\left(W_{1}, W_{2}\right)$ as referring to lianas and trees respectively. The covariate $X$ is the conductance to water $\left(\mathrm{H}_{2} \mathrm{O}\right)$ measured in mol $\mathrm{H}_{2} \mathrm{O} \mathrm{m}^{-2} \mathrm{~s}^{-1}$. Figure 1 presents estimates of the conditional Kendall's tau for both lianas (solid line) and trees (dashed line). For both species the dependencies range between negative values (for lower levels of conductance to water) to moderate positive values (for higher levels of conductance to water). The dependence is almost always stronger (in absolute value sense) for lianas than for trees. The data are available at https://doi.org/10.5479/data_stri/10088/30581.

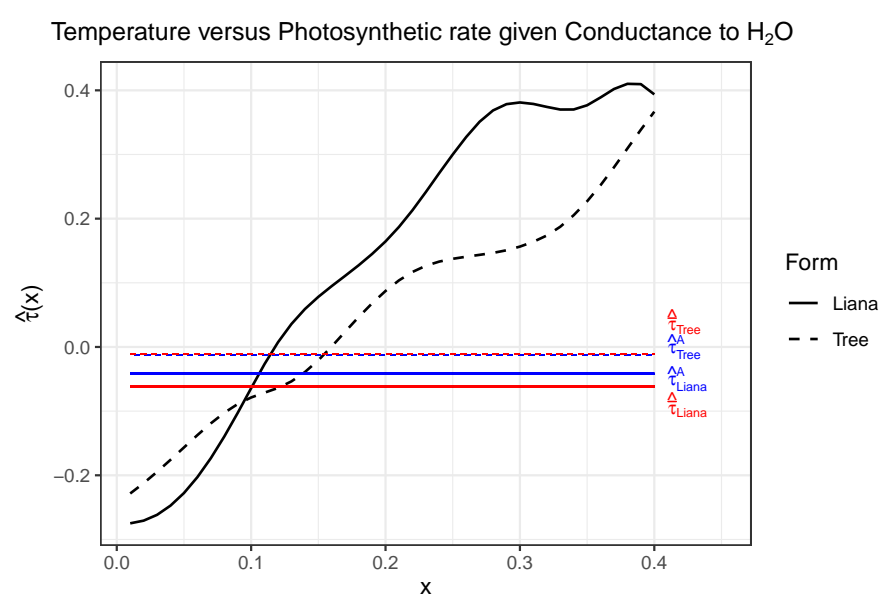

Figure 1: Lianas and trees. Estimated conditional Kendall's tau of the temperature and the photosynthetic rate given the conductance of water.

\subsection{Partial and average conditional Kendall's tau}

In order to study strengths of conditional dependencies of couples $\left(Y_{1}, Y_{2}\right)$ and $\left(Z_{1}, Z_{2}\right)$, when conditioning on the same covariate vector $\boldsymbol{X}$, an option would be to compare, for example, the conditional Kendall's tau functions $\tau_{Y_{1}, Y_{2}}(\boldsymbol{x})$ and $\tau_{Z_{1}, Z_{2}}(\boldsymbol{x})$, where the index refers to the couple for which the conditional measure holds. Comparing the $d$-variate functions is not an easy task. Since $\boldsymbol{X}$ is a random vector one could however look into an average value for the strength of the conditional dependence structure. In other words a possibility would be to consider the average conditional Kendall's tau, defined for a generic couple of random variables as,

$$
\mathrm{E}_{\boldsymbol{X}}[\tau(\boldsymbol{X})]
$$

In case of two couples $\left(Y_{1}, Y_{2}\right)$ and $\left(Z_{1}, Z_{2}\right)$ this would result into calculating $\mathrm{E}_{\boldsymbol{X}}\left[\tau_{Y_{1}, Y_{2}}(\boldsymbol{X})\right]$ and $\mathrm{E}_{\boldsymbol{X}}\left[\tau_{Z_{1}, Z_{2}}(\boldsymbol{X})\right]$. A larger value of, for example, $\mathrm{E}_{\boldsymbol{X}}\left[\tau_{Y_{1}, Y_{2}}(\boldsymbol{X})\right]$ would mean a larger, on average, conditional dependence between the components of the couple $\left(Y_{1}, Y_{2}\right)$ than between these of the couple $\left(Z_{1}, Z_{2}\right)$. In a similar way one can define other average conditional association measures, such as the average conditional Spearman's rho $\mathrm{E}_{\boldsymbol{X}}[\rho(\boldsymbol{X})]$. See for example [19].

For the illustration regarding photosynthesis in lianas and trees in Panamanian lowland tropical forests, the estimated values for the average conditional Kendall's tau (using the abbreviation $\tau^{\mathrm{A}}=\mathrm{E}_{\boldsymbol{X}}[\tau(\boldsymbol{X})]$ ) were $\widehat{\tau}_{\text {Liana }}^{\mathrm{A}}=-0.042$ and $\widehat{\tau}_{\text {Tree }}^{\mathrm{A}}=-0.011$. So an overall slighter stronger dependence in lianas than in trees. These estimates are depicted in Figure 1 as the (blue) horizontal lines.

The above concept of average conditional association measure is however not the only concept that could be useful when it comes to comparisons. One can also start by considering the average conditional copula $\mathrm{E}_{\boldsymbol{X}}\left[C_{\boldsymbol{X}}\left(u_{1}, u_{2}\right)\right]$ and Kendall's tau linked to this dependence function. As pointed out in the literature, see 
[19] and [32], the average conditional copula coincides for continuous random variables $Y_{1}$ and $Y_{2}$, with the concept of partial copula, which is the dependence structure between the two uniformized random variables $U_{1}=F_{1 X}\left(Y_{1}\right)$ and $U_{2}=F_{2 X}\left(Y_{2}\right)$, i.e.

$$
\mathrm{E}_{\boldsymbol{X}}\left[C_{\boldsymbol{X}}\left(u_{1}, u_{2}\right)\right]=P\left(U_{1} \leq u_{1}, U_{2} \leq u_{2}\right) \equiv \bar{C}\left(u_{1}, u_{2}\right) .
$$

Both the conditional copula and the partial copula are copula functions, i.e. satisfying all basic requirements for a function to be a copula. We next could also consider association measures that are resulting from this partial copula. We denote Kendall's tau of $\bar{C}$ as $\bar{\tau}$, the partial Kendall's tau. More precisely,

$$
\bar{\tau}=4 \iint \bar{C}\left(u_{1}, u_{2}\right) d \bar{C}\left(u_{1}, u_{2}\right)-1 .
$$

In general it holds that $\mathrm{E}_{\boldsymbol{X}}[\boldsymbol{\tau}(\boldsymbol{X})] \neq \overline{\boldsymbol{\tau}}$, as was also illustrated with examples in [19]. For other association measures such as Spearman's rho and Blomqvist's beta the average conditional and partial versions of them coincide. See [19] and [32].

In the illustration concerning photosynthesis in lianas and trees, we obtained estimated partial Kendall's tau values $\widehat{\bar{\tau}}_{\text {Liana }}=-0.062$ and $\widehat{\bar{\tau}}_{\text {Tree }}=-0.010$ for lianas and trees respectively. These values are also presented in Figure 1 by means of the (red) horizontal lines. Note that the difference between the two estimates is more pronounced for lianas than for trees. The estimated values for the average conditional Kendall's tau and the partial Kendall's tau for trees are almost equal.

From (1) it is seen that the influence of the realized covariate vector $\boldsymbol{x}$ in the conditional joint distribution function of $\left(Y_{1}, Y_{2}\right)$ given $\boldsymbol{X}=\boldsymbol{x}$ comes in at two separate levels: (i) on the level of the conditional dependence structure $C_{X}$; and (ii) on the level of the conditional marginal distribution functions $F_{1 X}$ and $F_{2 x}$. In applications one often makes the assumption that the conditional dependence structure is not influenced by the specific value $\boldsymbol{x}$ of the covariate $\boldsymbol{X}$, i.e. $H_{0}: C_{\boldsymbol{x}}\left(u_{1}, u_{2}\right)=C\left(u_{1}, u_{2}\right), \forall \boldsymbol{u}=\left(u_{1}, u_{2}\right)^{\mathrm{T}}$, and for all $\boldsymbol{x} \in \mathcal{R}_{\boldsymbol{X}}$. Such an assumption is termed as the simplifying assumption. See, for example, [22], [1], and [33]. Given the impact of such an assumption, quite some research in the past decade was devoted to developing tests for testing whether this simplified assumption holds or not. Among the more powerful tests are these based on comparing $\tau(\boldsymbol{x})$ with either $\mathrm{E}_{\boldsymbol{X}}[\boldsymbol{\tau}(\boldsymbol{X})]$ or $\bar{\tau}$, where under the null hypothesis $H_{0}$ the latter two quantities are equal. See, for example, [20], and also [8], and [18].

Given the importance and use of the concepts $\mathrm{E}_{\boldsymbol{X}}[\tau(\boldsymbol{X})]$ and $\bar{\tau}$, it is of interest to study them in detail, including addressing the questions: (i) how different can $\mathrm{E}_{\boldsymbol{X}}[\tau(\boldsymbol{X})]$ and $\bar{\tau}$ be?; (ii) what drives these differences?; (iii) can we provide precise conditions under which both quantities are equal? The aim of this paper is to provide answers to these questions.

\subsection{Further study in two settings}

Comparing $\mathrm{E}_{\boldsymbol{X}}[\boldsymbol{\tau}(\boldsymbol{X})]$ and $\bar{\tau}$ in full generality is not possible. However, we can study the two quantities under some sub-general settings. The following two settings are of particular interest.

SETTING 1 A setting of a mixture of two copulas:

$$
C_{\boldsymbol{x}}\left(u_{1}, u_{2}\right)=w(x) C_{1}\left(u_{1}, u_{2}\right)+(1-w(x)) C_{2}\left(u_{1}, u_{2}\right),
$$

where $w: \mathcal{R}_{\boldsymbol{X}} \rightarrow[0,1]$, is a weight function. Note that for a given $\boldsymbol{x} \in \mathcal{R}_{\boldsymbol{X}}, C_{\boldsymbol{X}}\left(u_{1}, u_{2}\right)$ is nothing but a linear combination of two copulas. As stated in [9, Theorem 1.4.5 on page 16], the set of all copulas is a convex set, and hence each linear combination of two copulas is again a copula. Hence $C_{\boldsymbol{x}}\left(u_{1}, u_{2}\right)$ in (2) is always a copula.

Setring 2 A trivariate Archimedean setting. In this it is assumed that the random triplet $\left(Y_{1}, Y_{2}, \boldsymbol{X}\right)$ has a joint distribution function $H$ and marginal distribution functions $F_{X}, F_{Y_{1}}$ and $F_{Y_{2}}$. In this setting we restrict to the univariate covariate case, i.e. $d=1$ in $\boldsymbol{X}=\left(X_{1}, \ldots, X_{d}\right)^{\mathrm{T}}$, denoting $\boldsymbol{X}=X$, since there is 
no multivariate equivalent to the probability integral transform that ensures that $F_{X}(X)$ has a uniform distribution. We have that

$$
H\left(x, y_{1}, y_{2}\right)=C\left(F_{X}(x), F_{Y_{1}}\left(y_{1}\right), F_{Y_{2}}\left(y_{2}\right)\right)
$$

where $C$ is a trivariate Archimedean copula, i.e.

$$
C\left(u_{1}, u_{2}, u_{3}\right)=\varphi^{-1}\left(\varphi\left(u_{1}\right)+\varphi\left(u_{2}\right)+\varphi\left(u_{3}\right)\right)
$$

with $\varphi:[0,1] \rightarrow \mathbb{R}_{+}$is the so-called generator which is such that $\varphi(1)=0, \lim _{u \rightarrow \infty} \varphi^{-1}(u)=0$ and further $\left(\varphi^{-1}\right)^{\prime}(t) \leq 0,\left(\varphi^{-1}\right)^{\prime \prime}(t) \geq 0$, and $\left(\varphi^{-1}\right)^{\prime \prime \prime}(t) \leq 0$.

We give some examples regarding these two settings.

\section{Example 2.1.}

Let $d=1$ and $w(x)=x$, with $x \in[0,1]$, and consider

$$
C_{x}\left(u_{1}, u_{2}\right)=x \Pi\left(u_{1}, u_{2}\right)+(1-x) M\left(u_{1}, u_{2}\right),
$$

which is a mixture of the independence copula $\Pi\left(u_{1}, u_{2}\right)=u_{1} u_{2}$, and the comonotonic copula $M\left(u_{1}, u_{2}\right)=$ $\min \left(u_{1}, u_{2}\right)$. Figure 2 shows a sample (of size 100) for this copula for three cases: when $x=0,0.5$ and $x=1$. With a value of the weight function $(w(x)=x)$ between 0 and 1 , one can cover a variety of dependence structures between comonotonicity (left) and independence (right).
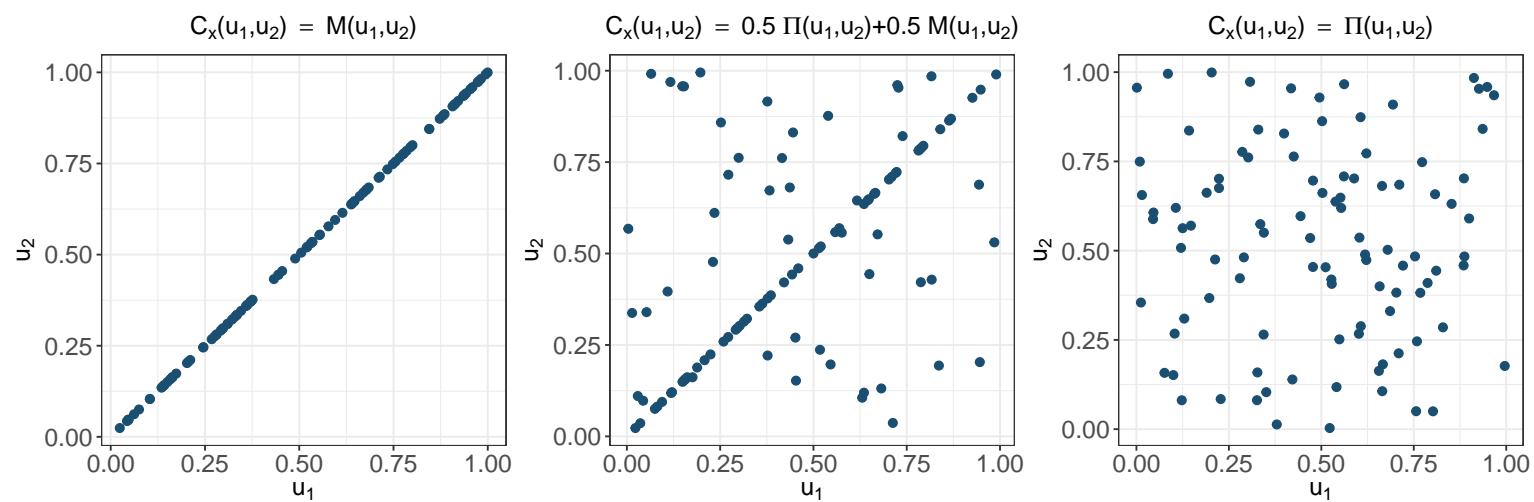

Figure 2: Scatterplots of a mixture of the independence copula $\Pi$ and the comonotonic copula $M$ for three values of $w(x)=x$ : $w(x)=0$ (left), $w(x)=0.5$ (middle), $w(x)=1$ (right).

\section{Example 2.2.}

Let $d=1$ and $C$ a Frank copula with generator as indicated in Table 2, and parameter $\theta=3$. Let $X$ be a random variable with a Beta $(1,3)$ distribution. Figure 3 presents contour plots of the unconditional copula of $\left(Y_{1}, Y_{2}\right)$, and of the conditional copula $C_{X}$ of $\left(Y_{1}, Y_{2}\right)$ for two realized values of $X$, namely $x=0.1$ and $x=0.7$. The values for the unconditional and conditional distributions are presented on the vertical axis on the right-hand side. As can be seen the contours of the unconditional copula (dotted curves) are clearly different from these of the two conditional copulas (solid and dashed curves) that also differ from each other. 


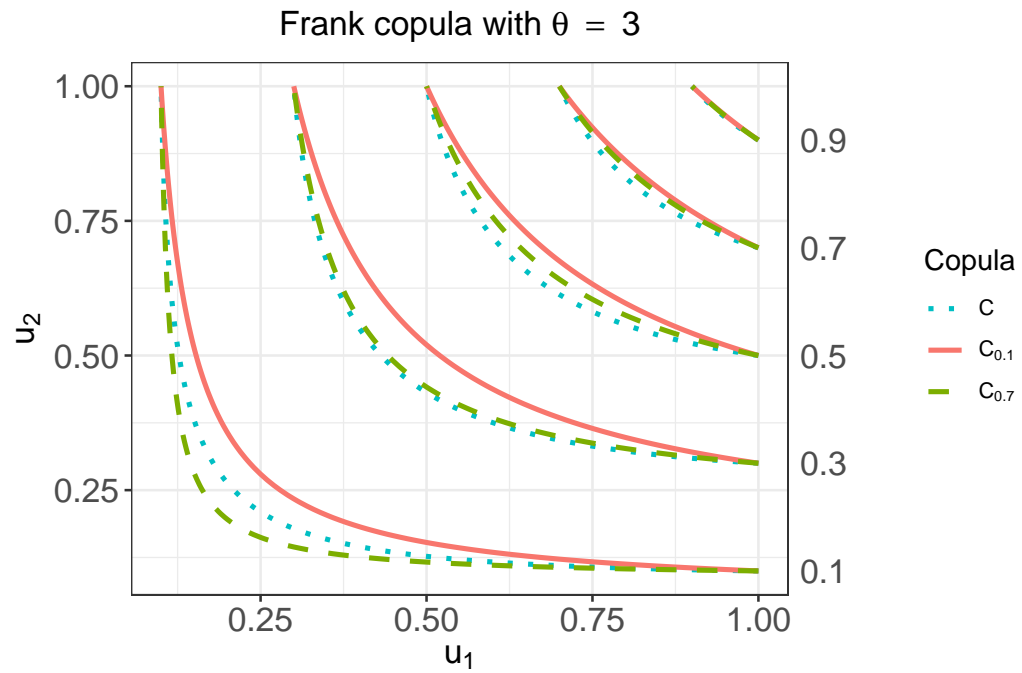

Figure 3: Contours of the unconditional and the conditional copula for $\left(Y_{1}, Y_{2}\right)$ in case of a trivariate Frank copula, and a marginal Beta $(1,3)$ distribution for $X$.

\section{Study in case of a mixture of copulas}

This section is devoted to the study of $\tau^{\mathrm{A}}=\mathrm{E}_{\boldsymbol{X}}[\tau(\boldsymbol{X})]$ and $\bar{\tau}$ in Setting 1, the setting of an arbitrary mixture of two copulas $C_{1}, C_{2}$ using a weight function $w(\cdot)$.

\subsection{Theoretical results}

A first result reads as follows.

Theorem 3.1. Suppose the conditional copula $C_{\boldsymbol{X}}$ is of the form (2). Denote $\mu_{j}=\mathrm{E}_{\boldsymbol{X}}\left[w^{j}(\boldsymbol{X})\right]$ for $j=1,2$. Then the following expressions hold:

(i). $\tau^{A}=4\left\{\mu_{2} \iint_{[0,1]^{2}} C_{1} d C_{1}+2\left(\mu_{1}-\mu_{2}\right) \iint_{[0,1]^{2}} C_{1} d C_{2}+\left(1-2 \mu_{1}+\mu_{2}\right) \iint_{[0,1]^{2}} C_{2} d C_{2}\right\}-1$;

(ii). $\bar{\tau}=4\left\{\mu_{1}^{2} \iint_{[0,1]^{2}} C_{1} d C_{1}+2 \mu_{1}\left(1-\mu_{1}\right) \iint_{[0,1]^{2}} C_{1} d C_{2}+\left(1-\mu_{1}\right)^{2} \iint_{[0,1]^{2}} C_{2} d C_{2}\right\}-1$;

(iii) $\bar{\tau}-\tau^{A}=4 \operatorname{Var}[w(\boldsymbol{X})]\left\{-\iint_{[0,1]^{2}} C_{1} d C_{1}+2 \iint_{[0,1]^{2}} C_{1} d C_{2}-\iint_{[0,1]^{2}} C_{2} d C_{2}\right\}$.

Theorem 3.1 has as immediate consequence the following corollary.

Corollary 3.1. If the conditional copula is of the form (2), then $\tau^{A}=\bar{\tau}$ provided that

$$
\text { or } \quad-\iint_{[0,1]^{2}} C_{1} d C_{1}+2 \iint_{[0,1]^{2}} C_{1} d C_{2}-\iint_{[0,1]^{2}} C_{2} d C_{2}=0 .
$$

From Corollary 3.1 it follows that for any covariate vector $\boldsymbol{X}$ with $\operatorname{Var}[w(\boldsymbol{X})]=0$ and/or for copulas $C_{1}$ and $C_{2}$ such that $T\left(C_{1}, C_{2}\right)=0$, with

$$
T\left(C_{1}, C_{2}\right)=-\iint_{[0,1]^{2}} C_{1} d C_{1}+2 \iint_{[0,1]^{2}} C_{1} d C_{2}-\iint_{[0,1]^{2}} C_{2} d C_{2},
$$


the concepts of average conditional Kendall's tau and partial Kendall's tau coincide.

A probabilistic interpretation of the quantity $T\left(C_{1}, C_{2}\right)$ is as follows. Denote by $\boldsymbol{V}=\left(V_{1}, V_{2}\right)$ (respectively $\left.\boldsymbol{Z}=\left(Z_{1}, Z_{2}\right)\right)$ a random couple, with uniform marginal distributions, having joint distribution $C_{1}$ (respectively $C_{2}$ ). Since $\iint_{[0,1]^{2}} C_{1} d C_{2}=\iint_{[0,1]^{2}} C_{2} d C_{1}$ (as can be deduced from [28, Corollary 5.1.2.]), it is seen that

$$
T\left(C_{1}, C_{2}\right)=\iint_{[0,1]^{2}}\left[C_{2}-C_{1}\right] d C_{1}-\iint_{[0,1]^{2}}\left[C_{2}-C_{1}\right] d C_{2},
$$

and thus $T\left(C_{1}, C_{2}\right)$ measures the difference between the two dependence structures, $C_{1}$ and $C_{2}$, under the two joint laws, that of $\boldsymbol{V}$ and that of $\boldsymbol{Z}$.

Theorem 3.2 establishes a sufficient and necessary condition for (4) to hold. Recall that for a copula $C$ Kendall's tau and Spearman's rho are given by

$$
\tau(C)=4 \iint_{[0,1]^{2}} C\left(u_{1}, u_{2}\right) d C\left(u_{1}, u_{2}\right)-1 \quad \text { and } \quad \rho(C)=12 \iint_{[0,1]^{2}} C\left(u_{1}, u_{2}\right) d \boldsymbol{u}-3 .
$$

Theorem 3.2. Suppose that the conditional copula $C_{\boldsymbol{X}}$ is of the form (2). Suppose that the range of $w(\boldsymbol{X})$ equals $[0,1]$ (and not a subset of it). Then, the following statements are equivalent

(i). $T\left(C_{1}, C_{2}\right)=0$;

(ii). For all $\gamma \in[0,1]$

$$
\tau\left(\gamma C_{1}+(1-\gamma) C_{2}\right)=\gamma \tau\left(C_{1}\right)+(1-\gamma) \tau\left(C_{2}\right)
$$

So, if it holds that Kendall's tau of any linear combination of $C_{1}$ and $C_{2}$ equals the linear combination of the Kendall's tau's of the two copulas, then we know from Corollary 3.1 that $\tau^{\mathrm{A}}=\bar{\tau}$.

When one of the two copulas, $C_{1}$ or $C_{2}$ in the mixture equals one of the Fréchet-Hoeffding bounds or the independence copula, we can provide easier equivalencies for $T\left(C_{1}, C_{2}\right)=0$. Recall that the lower FréchetHoeffding bound equals $W\left(u_{1}, u_{2}\right)=\max \left(u_{1}+u_{2}-1,0\right)$, and is called the countermonotonic copula; whereas the upper Fréchet-Hoeffding bound is $M\left(u_{1}, u_{2}\right)=\min \left(u_{1}, u_{2}\right)$ which is referred to as the comonotonic copula. For simplicity of presentation we present in Corollary 3.2 our findings when $C_{2}$ equals one of these 3 copulas $W, \Pi$ or $M$.

Corollary 3.2. Let the conditional copula $C_{X}$ be of the form (2), and $C$ any copula. Suppose that the range of $w(\boldsymbol{X})$ equals $[0,1]$ (and not a subset of it). Then the following statements hold.

1). If $C_{1}=C$, and $C_{2}=\Pi$, then (4) is equivalent to

$$
\tau(C)=\frac{2}{3} \rho(C)
$$

2). If $C_{1}=C$, and $C_{2}=M$, then (4) is equivalent to

$$
\int_{0}^{1} \delta(u) d u=\frac{\tau(C)+3}{8}
$$

where $\delta(u)=C(u, u)$ is the diagonal section of the copula $C$.

3). If $C_{1}=C$, and $C_{2}=W$, then (4) is equivalent to

$$
\int_{0}^{1} C(u, 1-u) d u=\frac{\tau(C)+1}{8}
$$

where $C(u, 1-u)$ is the opposite diagonal section of the copula $C$.

See [7], [6] and [12], among others, for recent work regarding diagonal and opposite diagonal sections of copulas. 


\subsection{Illustrative examples}

We next provide some examples that illustrate the use of Theorem 3.2, in the first set of examples, and Corollary 3.2, in a second set of examples. Additional examples can be found in Section S.1 of the Supplementary Material.

For convenience of the reader, we list in Table 1 some of the copulas that appear in the examples, with the ranges of the parameters and the expressions for Kendall's tau and Spearman's rho. See [28] for derivation of these results.

Table 1: Some copulas.

\begin{tabular}{l|l|l}
\hline Copula & Parameter restrictions & $\begin{array}{l}\text { Kendall's tau and } \\
\text { Spearman's rho }\end{array}$ \\
\hline Farlie-Gumbel-Morgenstein (FGM) & $\theta \in[-1,1]$ & $\tau(C)=\frac{2 \theta}{9}$ \\
$C\left(u_{1}, u_{2}\right)=u_{1} u_{2}+\theta u_{1} u_{2}\left(1-u_{1}\right)\left(1-u_{2}\right)$ & & $\rho(C)=\frac{\theta}{3}$ \\
\hline Fréchet & $\alpha, \beta \in[0,1]$, & $\tau(C)=\frac{(\alpha-\beta)(\alpha+\beta+2)}{3}$ \\
$C\left(u_{1}, u_{2}\right)=\alpha M\left(u_{1}, u_{2}\right)$ & $\alpha+\beta \leq 1$ & $\rho(C)=\alpha-\beta$ \\
$+(1-\alpha-\beta) \Pi\left(u_{1}, u_{2}\right)+\beta W\left(u_{1}, u_{2}\right)$ & & \\
\hline
\end{tabular}

\section{Example 3.1.}

Consider $C_{1}$ and $C_{2}$ both members of the Farlie-Gumbel-Morgenstern (FGM) copula family, i.e. $C_{1}$ and $C_{2}$ are of the form

$$
C_{j}\left(u_{1}, u_{2}\right)=u_{1} u_{2}+\theta_{j} u_{1} u_{2}\left(1-u_{1}\right)\left(1-u_{2}\right), \quad \text { with } \theta_{j} \in[-1,1], \quad j=1,2 \text {. }
$$

Note that when $\theta_{j}=0$, we get that $C_{j}=\Pi$, the independence copula.

We look at an arbitrary linear combination of two arbitrary FGM copulas, and find

$$
\begin{aligned}
C\left(u_{1}, u_{2}\right) & =\gamma C_{1}\left(u_{1}, u_{2}\right)+(1-\gamma) C_{2}\left(u_{1}, u_{2}\right) \\
& =u_{1} u_{2}+\gamma \theta_{1} u_{1} u_{2}\left(1-u_{1}\right)\left(1-u_{2}\right)+(1-\gamma) \theta_{2} u_{1} u_{2}\left(1-u_{1}\right)\left(1-u_{2}\right) \\
& =u_{1} u_{2}+\left[\gamma \theta_{1}+(1-\gamma) \theta_{2}\right] u_{1} u_{2}\left(1-u_{1}\right)\left(1-u_{2}\right) .
\end{aligned}
$$

A first question to answer is whether such an arbitrary linear combination of FGM copulas is again a FGM copula. To answer this question we need to check whether

$$
\gamma \theta_{1}+(1-\gamma) \theta_{2} \in[-1,1]
$$

Noting that

$$
-1=\gamma(-1)+(1-\gamma)(-1) \leq \gamma \theta_{1}+(1-\gamma) \theta_{2} \leq \gamma 1+(1-\gamma) 1=1,
$$

it immediately follows that (12) holds. Hence an arbitrary linear combination $\gamma C_{1}+(1-\gamma) C_{2}$ of two FarlieGumbel-Morgenstern copulas is again a Farlie-Gumbel-Morgenstern copula. Consequently, Kendall's tau of such a linear combination equals (see Table 1)

$$
\tau(C)=\frac{2}{9}\left[\gamma \theta_{1}+(1-\gamma) \theta_{2}\right]=\gamma \tau\left(C_{1}\right)+(1-\gamma) \tau\left(C_{2}\right)
$$


for any $\gamma \in[0,1]$ and hence Theorem 3.2(ii) is satisfied and consequently $\bar{\tau}=\tau^{\mathrm{A}}$.

\section{Example 3.2.}

Consider $C_{1}$ and $C_{2}$ both members of the Fréchet family of copulas, i.e.

$$
C_{j}\left(u_{1}, u_{2}\right)=\alpha_{j} M\left(u_{1}, u_{2}\right)+\left(1-\alpha_{j}-\beta_{j}\right) \Pi\left(u_{1}, u_{2}\right)+\beta_{j} W\left(u_{1}, u_{2}\right) \quad \text { for } j=1,2,
$$

with $\alpha_{1}, \alpha_{2}, \beta_{1}, \beta_{2} \in[0,1]$ and such that $\alpha_{1}+\beta_{1} \leq 1$ and $\alpha_{2}+\beta_{2} \leq 1$.

Consider a copula $C$ that is a linear combination of two arbitrary Fréchet copulas. We find

$$
\begin{aligned}
& C\left(u_{1}, u_{2}\right) \\
& =\gamma C_{1}\left(u_{1}, u_{2}\right)+(1-\gamma) C_{2}\left(u_{1}, u_{2}\right) \\
& =\gamma\left(\alpha_{1} M\left(u_{1}, u_{2}\right)+\left(1-\alpha_{1}-\beta_{1}\right) \Pi\left(u_{1}, u_{2}\right)+\beta_{1} W\left(u_{1}, u_{2}\right)\right)+(1-\gamma)\left(\alpha_{2} M\left(u_{1}, u_{2}\right)\right. \\
& \left.\quad+\left(1-\alpha_{2}-\beta_{2}\right) \Pi\left(u_{1}, u_{2}\right)+\beta_{2} W\left(u_{1}, u_{2}\right)\right) \\
& =\left[\gamma \alpha_{1}+(1-\gamma) \alpha_{2}\right] M\left(u_{1}, u_{2}\right)+\left[1-\gamma\left(\alpha_{1}+\beta_{1}\right)-(1-\gamma)\left(\alpha_{2}+\beta_{2}\right)\right] \Pi\left(u_{1}, u_{2}\right) \\
& \quad+\left[\gamma \beta_{1}+(1-\gamma) \beta_{2}\right] W\left(u_{1}, u_{2}\right) .
\end{aligned}
$$

Hence such a linear combination leads again to a linear combination of the three basic copulas $\Pi, M$ and $W$. Furthermore, this linear combination is again a Fréchet copula as is easily seen by defining $\alpha=\gamma \alpha_{1}+(1-\gamma) \alpha_{2}$ and $\beta=\gamma \beta_{1}+(1-\gamma) \beta_{2}$, which satisfy $\alpha, \beta \in[0,1]$, and noting that

$$
\alpha+\beta=\gamma\left(\alpha_{1}+\beta_{1}\right)+(1-\gamma)\left(\alpha_{2}+\beta_{2}\right) \leq \max \left(\alpha_{1}+\beta_{1}, \alpha_{2}+\beta_{2}\right) \leq 1 .
$$

Hence, Kendall's tau for the arbitrary linear combination of $C_{1}$ and $C_{2}$ is given by (see Table 1)

$$
\begin{aligned}
\tau(C) & =\frac{1}{3}\left\{\left(\gamma \alpha_{1}+(1-\gamma) \alpha_{2}-\gamma \beta_{1}-(1-\gamma) \beta_{2}\right) \times\left(\gamma \alpha_{1}+(1-\gamma) \alpha_{2}+\gamma \beta_{1}+(1-\gamma) \beta_{2}+2\right)\right\} \\
& =\frac{1}{3}\left\{\left(\gamma\left(\alpha_{1}-\beta_{1}\right)+(1-\gamma)\left(\alpha_{2}-\beta_{2}\right)\right) \times\left(\gamma\left(\alpha_{1}+\beta_{1}\right)+(1-\gamma)\left(\alpha_{2}+\beta_{2}\right)+2\right)\right\} .
\end{aligned}
$$

Noting that

$$
\gamma\left(\alpha_{1}+\beta_{1}\right)+(1-\gamma)\left(\alpha_{2}+\beta_{2}\right)+2=\gamma\left(\alpha_{1}+\beta_{1}+2\right)+(1-\gamma)\left(\alpha_{2}+\beta_{2}+2\right)
$$

we get

$$
\begin{aligned}
\tau(C)= & \frac{1}{3}\left\{\left(\gamma\left(\alpha_{1}-\beta_{1}\right)+(1-\gamma)\left(\alpha_{2}-\beta_{2}\right)\right) \times\left(\gamma\left(\alpha_{1}+\beta_{1}+2\right)+(1-\gamma)\left(\alpha_{2}+\beta_{2}+2\right)\right)\right\} \\
= & \gamma^{2} \frac{1}{3}\left(\alpha_{1}-\beta_{1}\right)\left(\alpha_{1}+\beta_{1}+2\right)+(1-\gamma)^{2} \frac{1}{3}\left(\alpha_{2}-\beta_{2}\right)\left(\alpha_{2}+\beta_{2}+2\right) \\
& \quad+\frac{1}{3} \gamma(1-\gamma)\left[\left(\alpha_{1}-\beta_{1}\right)\left(\alpha_{2}+\beta_{2}+2\right)+\left(\alpha_{2}-\beta_{2}\right)\left(\alpha_{1}+\beta_{1}+2\right)\right] \\
= & \gamma^{2} \tau\left(C_{1}\right)+(1-\gamma)^{2} \tau\left(C_{2}\right)+\frac{1}{3} \gamma(1-\gamma)\left[\left(\alpha_{1}-\beta_{1}\right)\left(\alpha_{2}+\beta_{2}+2\right)+\left(\alpha_{2}-\beta_{2}\right)\left(\alpha_{1}+\beta_{1}+2\right)\right] .
\end{aligned}
$$

We need to study the last term a bit more closely. First of all note that

$$
\begin{aligned}
& \left(\alpha_{1}-\beta_{1}\right)\left(\alpha_{2}+\beta_{2}+2\right)+\left(\alpha_{2}-\beta_{2}\right)\left(\alpha_{1}+\beta_{1}+2\right) \\
= & \left(\alpha_{1}-\beta_{1}\right)\left(\alpha_{1}+\beta_{1}+2\right)+\left(\alpha_{2}-\beta_{2}\right)\left(\alpha_{2}+\beta_{2}+2\right) \\
& \quad+\left[\left(\alpha_{2}-\alpha_{1}\right)+\left(\beta_{2}-\beta_{1}\right)\right]\left[\left(\beta_{2}-\beta_{1}\right)-\left(\alpha_{2}-\alpha_{1}\right)\right] \\
= & \left(\alpha_{1}-\beta_{1}\right)\left(\alpha_{1}+\beta_{1}+2\right)+\left(\alpha_{2}-\beta_{2}\right)\left(\alpha_{2}+\beta_{2}+2\right)+\left(\beta_{2}-\beta_{1}\right)^{2}-\left(\alpha_{2}-\alpha_{1}\right)^{2},
\end{aligned}
$$


and hence

$$
\begin{aligned}
\tau(C)= & \gamma^{2} \tau\left(C_{1}\right)+(1-\gamma)^{2} \tau\left(C_{2}\right) \\
& +\frac{1}{3} \gamma(1-\gamma)\left[\left(\alpha_{1}-\beta_{1}\right)\left(\alpha_{1}+\beta_{1}+2\right)+\left(\alpha_{2}-\beta_{2}\right)\left(\alpha_{2}+\beta_{2}+2\right)+\left(\beta_{2}-\beta_{1}\right)^{2}-\left(\alpha_{2}-\alpha_{1}\right)^{2}\right] \\
= & \gamma^{2} \tau\left(C_{1}\right)+(1-\gamma)^{2} \tau\left(C_{2}\right)+\gamma(1-\gamma)\left\{\tau\left(C_{1}\right)+\tau\left(C_{2}\right)+\frac{1}{3}\left[\left(\beta_{2}-\beta_{1}\right)^{2}-\left(\alpha_{2}-\alpha_{1}\right)^{2}\right]\right\} \\
= & \gamma \tau\left(C_{1}\right)+(1-\gamma) \tau\left(C_{2}\right)+\gamma(1-\gamma) \frac{1}{3}\left[\left(\beta_{2}-\beta_{1}\right)^{2}-\left(\alpha_{2}-\alpha_{1}\right)^{2}\right] .
\end{aligned}
$$

From this it is easily seen that (7) holds if and only if

$$
\left(\beta_{2}-\beta_{1}\right)^{2}=\left(\alpha_{2}-\alpha_{1}\right)^{2},
$$

and hence

$$
\bar{\tau}=\tau^{\mathrm{A}} \Longleftrightarrow\left(\alpha_{1}-\alpha_{2}\right)^{2}=\left(\beta_{1}-\beta_{2}\right)^{2},
$$

i.e. when either $\alpha_{1}-\alpha_{2}=\beta_{1}-\beta_{2}$ or $\alpha_{1}-\alpha_{2}=\beta_{2}-\beta_{1}$.

In Example S.1 in Section S.1 in the Supplementary Material we illustrate Corollary 3.2 via some special cases of this example.

\section{Example 3.3.}

Let $C_{2}=\Pi$ and consider $C$ a copula of the form

$$
C\left(u_{1}, u_{2}\right)=u_{1} u_{2}+f\left(u_{1}\right) g\left(u_{2}\right),
$$

where the functions $f$ and $g$ are two functions from $[0,1] \rightarrow \mathbb{R}$, satisfying the following conditions:

(1). $f(0)=f(1)=g(0)=g(1)=0$;

(2). $f$ and $g$ are absolutely continuous;

(3). denoting the sets $A=\left\{u \in[0,1]: f^{\prime}(u)\right.$ exists $\}$ and $B=\left\{u \in[0,1]: g^{\prime}(u)\right.$ exists $\}$, and putting $\alpha_{f}=$ $\inf \left\{f^{\prime}(u): u \in A\right\}, \beta_{f}=\sup \left\{f^{\prime}(u): u \in A\right\}$, and similarly $\alpha_{g}=\inf \left\{g^{\prime}(u): u \in B\right\}, \beta_{g}=\sup \left\{f^{\prime}(u): u \in\right.$ $B\}$, then it must hold that $\min \left(\alpha_{f} \alpha_{g}, \beta_{f} \beta_{g}\right) \geq-1$.

It was shown in [30] that if $f$ and $g$ satisfy the above conditions then $C$ is an absolutely continuous copula. In addition [30, Theorem 3.2, page 320] proved that for $C$ in (15) statement (8) holds. See also [13, page 2148].

\section{Example 3.4.}

Let $C_{2}=M$ and let $C$ be a Fredricks-Nelson diagonal copula. See [28, page 85]. For given $\delta:[0,1] \mapsto[0,1]$ which maps $t$ into $\delta(t)$, and satisfies

$$
\begin{aligned}
& \delta(1)=1 \\
& \forall t_{1}, t_{2} \in[0,1], \text { with } t_{1} \leq t_{2}: 0 \leq \delta\left(t_{2}\right)-\delta\left(t_{1}\right) \leq 2\left(t_{2}-t_{1}\right) \\
& \forall t \in[0,1]: \delta(t) \leq t,
\end{aligned}
$$

a Fredricks-Nelson diagonal copula is defined as

$$
C\left(u_{1}, u_{2}\right)=\min \left(u_{1}, u_{2}, 0.5\left[\delta\left(u_{1}\right)+\delta\left(u_{2}\right)\right]\right) .
$$

The function $C$ is a copula with diagonal section $\delta(t)$.

Do such kind of copulas satisfy equality (9)? According to [28, page 166] Kendall's tau for such a copula equals

$$
\tau(C)=4 \int_{0}^{1} \delta(u) d u-1,
$$


and hence, in general, for a Fredricks-Nelson diagonal copula

$$
\int_{0}^{1} \delta(u) d u=\frac{\tau(C)+1}{4} \neq \frac{\tau(C)+3}{8} .
$$

The only case in which there is equality is when

$$
\frac{\tau(C)+1}{4}=\frac{\tau(C)+3}{8} \Longleftrightarrow 2(\tau(C)+1)=\tau(C)+3 \Longleftrightarrow \tau(C)=1
$$

and thus

$$
\int_{0}^{1} \delta(u) d u=\frac{1}{2} .
$$

A copula $C$ for which this holds is the copula $C=M$, leading to $T(M, M)=0$.

\section{Study in case of an Archimedean copula setting}

We next turn to the study of $\mathrm{E}_{X}[\tau(X)]$ and $\bar{\tau}$ in Setting 2 in Section 2.3. Firstly it is important to remark that when the copula of $\left(Y_{1}, Y_{2}, X\right)$ is an Archimedean copula, then the conditional copula function is again an Archimedean copula for which the generator has a known expression. For convenience of the reader we state this result of [25] in Lemma 4.1.

Lemma 4.1 ([25]). If the copula of $\left(Y_{1}, Y_{2}, X\right)$ is an Archimedean copula with generator $\varphi$, then, for any marginal $X$, the conditional copula of $\left(Y_{1}, Y_{2}\right)$ given $X=x$, denoted by $C_{X}$ is also an Archimedean copula with generator

$$
\varphi_{X}(t)=\varphi\left[\left(\varphi^{\prime}\right)^{-1}\left(\frac{\varphi^{\prime}\left(F_{X}(x)\right)}{t}\right)\right]-\varphi\left(F_{X}(x)\right) .
$$

If moreover the generator $\varphi$ satisfies

$$
\varphi^{\prime}(t) \varphi^{\prime \prime \prime}(t) \geq\left[\varphi^{\prime \prime}(t)\right]^{2}, \quad \forall 0 \leq t \leq 1,
$$

the conditional copula $C_{\varphi_{x}}$ of the conditional distribution of $\left(Y_{1}, Y_{2}\right)$ given $X=x$ is bounded from above by

$$
M_{1}\left(u_{1}, u_{2}\right)=\frac{u_{1} u_{2}}{u_{1}+u_{2}-u_{1} u_{2}},
$$

i.e. $C_{\varphi_{x}}\left(u_{1}, u_{2}\right) \leq M_{1}\left(u_{1}, u_{2}\right), \quad \forall\left(u_{1}, u_{2}\right) \in[0,1]^{2}$.

\section{Remark 4.1.}

1. The conditional copula $C_{\varphi_{x}}$, defined as in (3), with generator $\varphi_{x}$ does not necessarily belong to the same Archimedean copula family as the random vector $\left(Y_{1}, Y_{2}, X\right)$, as pointed out already in [25]. This fact is also clear from some of the illustrative examples in Section 4.2.

2. The upperbound copula $M_{1}$ for the conditional copula $C_{x}$ plays a role similar to that of the FréchetHoeffding upperbound for unconditional copulas. As remarked by [25], Kendall's tau for $M_{1}$ equals 1/3, which is thus an upperbound for Kendall's tau associated to the conditional copula $C_{x}$.

\subsection{Theoretical results}

Before stating the main theoretical result for this setting, in Theorem 4.1, we introduce some notations. The first order partial derivative of the conditional copula $C_{x}$ with generator as in (16) is given by

$$
g\left(w, u_{1}, u_{2}\right)=: \frac{\partial C_{x}\left(u_{1}, u_{2}\right)}{\partial u_{1}}=g_{1}\left(w, u_{1}\right) h\left(w, u_{1}, u_{2}\right),
$$


where we denoted $w=F_{X}(x)$, and

$$
g_{1}\left(w, u_{1}\right)=\frac{1}{u_{1}^{3} \varphi^{\prime \prime}\left(\left(\varphi^{\prime}\right)^{-1}\left(\frac{\varphi^{\prime}(w)}{u_{1}}\right)\right)} \quad \text { and } h\left(w, u_{1}, u_{2}\right)=\left(\varphi^{\prime}(w)\right)^{3} \frac{\varphi^{\prime \prime}\left(f\left(w, u_{1}, u_{2}\right)\right)}{\left[\varphi^{\prime}\left(f\left(w, u_{1}, u_{2}\right)\right)\right]^{3}},
$$

wherein

$$
f\left(w, u_{1}, u_{2}\right)=\varphi^{-1}\left[\varphi\left(\left(\varphi^{\prime}\right)^{-1}\left(\frac{\varphi^{\prime}(w)}{u_{1}}\right)\right)+\varphi\left(\left(\varphi^{\prime}\right)^{-1}\left(\frac{\varphi^{\prime}(w)}{u_{2}}\right)\right)-\varphi(w)\right] .
$$

Note that the function $f\left(w, u_{1}, u_{2}\right)$ is symmetric in the arguments $\left(u_{1}, u_{2}\right)$, and hence the same holds for the function $h\left(w, u_{1}, u_{2}\right)$.

Theorem 4.1. If the copula of $\left(Y_{1}, Y_{2}, X\right)$ is an Archimedean copula with generator $\varphi$, then

$$
\begin{aligned}
& \tau^{A}=1-4 \iint_{[0,1]^{2}}\left\{\int_{[0,1]} g\left(w, u_{1}, u_{2}\right) g\left(w, u_{2}, u_{1}\right) d w\right\} d u_{1} d u_{2} \\
& \bar{\tau}=1-4 \iint_{[0,1]^{2}}\left\{\int_{[0,1]} g\left(w, u_{1}, u_{2}\right) d w\right\}\left\{\int_{[0,1]} g\left(w, u_{2}, u_{1}\right) d w\right\} d u_{1} d u_{2} .
\end{aligned}
$$

A sufficient condition for $\tau^{A}$ and $\bar{\tau}$ being equal is thus: $\forall\left(u_{1}, u_{2}\right) \in[0,1]^{2}$ it holds that

$$
\left\{\int_{[0,1]} g\left(w, u_{1}, u_{2}\right) d w\right\}\left\{\int_{[0,1]} g\left(w, u_{2}, u_{1}\right) d w\right\}=\int_{[0,1]} g\left(w, u_{1}, u_{2}\right) g\left(w, u_{2}, u_{1}\right) d w .
$$

Furthermore, an upperbound for $\left|\tau^{A}-\bar{\tau}\right|$ is

$$
\left.4 \iint_{[0,1]^{2}} \mid \int_{[0,1]} g\left(w, u_{1}, u_{2}\right) d w\right\}\left\{\int_{[0,1]} g\left(w, u_{2}, u_{1}\right) d w\right\}-\left\{\int_{[0,1]} g\left(w, u_{1}, u_{2}\right) g\left(w, u_{2}, u_{1}\right) d w\right\} \mid d u_{1} d u_{2} .
$$

If in addition the generator satisfies Condition (17) then both $\tau^{A}$ and $\bar{\tau}$ are bounded above by $1 / 3$.

The proof of Theorem 4.1 is provided in Appendix A, and relies on Lemma 4.1.

\section{Remark 4.2.}

Statements (18) and (19) in fact hold in general, with the function $g\left(w, u_{1}, u_{2}\right)$ being the first order partial derivative of the conditional copula with respect to its first argument. Similarly to what is known for the first order partial derivative for an unconditional copula (see for example [28, expression (2.9.1), page 41]), the first order partial derivative $g\left(w, u_{1}, u_{2}\right)$ of the conditional copula $C_{X}\left(u_{1}, u_{2}\right)$ can be interpreted as follows. This first order partial derivative is the conditional probability $P\left(U_{2} \leq u_{2} \mid U_{1}=u_{1}, X=x\right)=P\left(U_{2} \leq u_{2} \mid U_{1}=\right.$ $\left.u_{1}, F_{X}(X)=w\right)$. Condition (19), is not easy to check, unless in some specific cases. For example, in case the simplifying assumption holds (i.e. $P\left(U_{1} \leq u_{1}, U_{2} \leq u_{2} \mid X=x\right)=P\left(U_{1} \leq u_{1}, U_{2} \leq u_{2}\right)$, for all $\left.\left(u_{1}, u_{2}\right)\right)$, then statement (19) holds, since the integrals over $w$ in (19) disappear. In case of the Archimedean copula setting, this happens when the characterization of the generator of $C_{X}$ is not influenced by the value of $w=F_{X}(x)$. This is for example the case when $C$ is a Clayton copula. See further Section 4.2.

\subsection{Illustrative examples}

The following lemma, from [28, Theorem 4.1.5 (3) on page 113], states that a generator of an Archimedean copula is an equivalence class, in the sense that if we multiply a generator with a positive constant, it still generates the same copula. This result is useful in the examples. 
Lemma $4.2([28])$. Let $C$ be an Archimedean copula with generator $\varphi$. Then $\widetilde{\varphi}(t)=c \varphi(t)$, with $c>0$ any constant, is also a generator of $C$.

Table 2: Some Archimedean copulas and their generators.

\begin{tabular}{|c|c|c|}
\hline $\begin{array}{l}\text { Archimedean } \\
\text { family }\end{array}$ & $\begin{array}{l}\text { Generator } \\
\varphi_{\theta}(t)\end{array}$ & $\begin{array}{l}\text { Parameter } \\
\text { restrictions }\end{array}$ \\
\hline Clayton & $\frac{1}{\theta}\left(t^{-\theta}-1\right)$ & $\theta>0$ \\
\hline Frank & $-\ln \frac{e^{-\theta t}-1}{e^{-\theta}-1}$ & $\theta \in \mathbb{R}_{0}$ \\
\hline Ali-Mikhail-Haq & $\ln \frac{1-\theta(1-t)}{t}$ & $\theta \in[-1,1) \backslash\{0\}$ \\
\hline Gumbel-Hougaard & $(-\ln t)^{\theta}$ & $\theta \geq 1$ \\
\hline $\begin{array}{l}\text { Copula family }(4.2 .7) \text { in } \\
\text { [28, Table 4.1, Section 4.2] } \\
\text { (Copula 1, hereafter) }\end{array}$ & $-\ln (\theta t+(1-\theta))$ & $\theta \in(0,1]$ \\
\hline $\begin{array}{l}\text { Copula family (4.2.16) in [28, Table 4.1] } \\
\text { (Copula 2, hereafter) }\end{array}$ & $\left(\frac{\theta}{t}+1\right)(1-t)$ & $\theta \geq 0$ \\
\hline $\begin{array}{l}\text { Copula family }(4.2 .2) \text { in }[28, \text { Table } 4.1] \\
\text { (Copula 3, hereafter) }\end{array}$ & $(1-t)^{\theta}$ & $\theta>1$ \\
\hline
\end{tabular}

In Table 2 we list a few examples of Archimedean copulas, with their generator, as well as the restrictions on the parameter space (ensuring also that all further expressions exist). We are interested in finding the conditional copula $C_{X}$ for these examples. Given expression (16) it suffices to calculate, for a specific example, the derivative of the generator and its inverse. From these it is straightforward to obtain the expression for the generator of the conditional copula. For convenience we list in Table 4 these expressions, and in Table 3 we provide the generators $\varphi_{x}(\cdot)$ of the associated conditional copula $C_{x}$, and comment if possible on the Archimedean family to which it belongs.

The examples of Clayton, Frank and Ali-Mikhail-Haq (AHM) copula were already mentioned in [25], although the precise expression for the generator for the conditional copula was not provided for the AliMikhail-Haq copula. Some important general remarks can be made based on the considered examples. First, note that Lemma 4.2 is needed for concluding that the conditional copula resulting from a Clayton copula is again a Clayton copula. Moreover the parameter for this latter Clayton copula does not depend on $F_{X}(x)$, and hence the simplifying assumption holds for this specific example. Second, the influence of $X$ and its realized value $x$ is transparant through the value $w=F_{X}(x)$. Note that in the case of Frank copula, the conditional copula is an Ali-Mikhail-Haq copula provided the restriction holds, i.e. the parameter space determined by $-\ln 2 \leq \theta F_{X}(x)$ depends on this realized value $x$. In other words the range of $x$ values that gives a valid conditional copula depends on the given value of $\theta$. We illustrate this further in Example 4.1 below.

Example 4.1 (Frank copula). 
Table 3: Some Archimedean copulas and the associated generators for $C_{\chi}$. Herein $w=F_{X}(x)$.

\begin{tabular}{l|l|l}
\hline $\begin{array}{l}\text { Archimedean } \\
\text { family }\end{array}$ & $\begin{array}{l}\text { Generator of } C_{X} \\
\varphi_{\theta, x}(t)\end{array}$ & $\begin{array}{l}\text { Conditional copula type } \\
\text { restriction }\end{array}$ \\
\hline Clayton & $\frac{w^{-\theta}}{\theta+1} \cdot \frac{1}{r(\theta)}\left(t^{-r(\theta)}-1\right)$ with $r(\theta)=\frac{\theta}{\theta+1}$ & Clayton, if $w \neq 0$ \\
Frank & $\ln \left(\frac{1-p_{x}(\theta)(1-t)}{t}\right)$ & Ali-Mikhail-Haq \\
& $p_{x}(\theta)=1-e^{-\theta w}$ & $-\ln (2) \leq \theta F_{X}(x)$ \\
Ali-Mikhail-Haq & $\ln \left(\frac{\left(1-\theta+\sqrt{(1-\theta)^{2}+4 \theta t w(1-\theta+\theta w)}\right)^{2}}{4 t(1-\theta+\theta w)^{2}}\right)$ & no specific name \\
Copula 1 & $-\ln (t)$ & $\begin{array}{l}\text { Gumbel-Hougaard copula, } \\
\text { with parameter 1 }\end{array}$ \\
Copula 2 & $\theta\left(\left(\sqrt{\frac{\theta t}{\theta w^{-2}+1-t}}\right)^{-1}-w^{-1}\right)-\left(\sqrt{\frac{\theta t}{\theta w^{-2}+1-t}}-w\right)$ & no specific name \\
Copula 3 & $(1-w)^{\theta}\left(t^{\theta /(1-\theta)}-1\right)$ & $\begin{array}{l}\text { Clayton, if } w \neq 1 \\
\text { with parameter } \frac{\theta}{\theta-1}\end{array}$ \\
\hline
\end{tabular}

Table 4: Some Archimedean copulas, the derivative of the generator and its inverse.

\begin{tabular}{l|l|l}
\hline $\begin{array}{l}\text { Archimedean } \\
\text { family }\end{array}$ & $\begin{array}{l}\text { Derivative generator } \\
\varphi_{\theta}^{\prime}(t)\end{array}$ & $\begin{array}{l}\text { Inverse of the derivative } \\
\left(\varphi_{\theta}^{\prime}\right)^{-1}(s)\end{array}$ \\
\hline Clayton & $-t^{-(\theta+1)}$ & $(-s)^{-1 /(\theta+1)}$ \\
Frank & $\frac{\theta e^{-\theta t}}{e^{-\theta t}-1}$ & $-\frac{1}{\theta} \ln \left(\frac{s}{s-\theta}\right)$ \\
Ali-Mikhail-Haq & $\frac{\theta-1}{t(1-\theta+\theta t)}$ & $\frac{\theta-1+\sqrt{(1-\theta)^{2}-\frac{4 \theta(1-\theta)}{s}}}{2 \theta}$ \\
Copula 1 & $\frac{-\theta}{\theta t+1-\theta}$ & $\frac{1}{\theta}\left(\frac{-\theta}{s}-1+\theta\right)$ \\
Copula 2 & $-\frac{\theta}{t^{2}}-1$ & $\sqrt{\frac{-\theta}{1+s}}$ \\
Copula 3 & $-\theta(1-t)^{\theta-1}$ & $1-\left(\frac{-s}{\theta}\right)^{1 /(\theta-1)}$ \\
\hline
\end{tabular}

As is seen from Table 3 the conditional copula is an Ali-Mikhail-Haq copula, where the parameter $\theta$ needs to satisfy $-\ln (2) \leq \theta F_{X}(x)$. To provide some further insights we look into the conditional Kendall's tau associated to the resulting AMH copula, which equals

$$
\tau(x)=1-\frac{2}{3} \frac{p_{x}(\theta)+\left(1-p_{x}(\theta)\right)^{2} \ln \left(1-p_{x}(\theta)\right)}{\left(p_{x}(\theta)\right)^{2}},
$$

with $p_{x}(\theta)$ as in Table 3. For $d=1$ and for a given value $\theta \geq 0$ the constraint $-\ln (2) \leq \theta F_{X}(x)$ is satisfied for all $x$. For $\theta<0$ this constraint leads to the restriction that $F_{X}(x) \leq-\theta^{-1} \ln (2)$. Figures 4 and 5 depict the conditional Kendall's tau together with the average conditional Kendall's tau, for two different marginal 
distributions: (i) $X \sim \mathrm{U}[a, b]$ (Figure 4); and (ii) $X \sim \operatorname{Beta}(c, d)$ (Figure 5). To illustrate further the influence of the marginal distributions, we considered three different settings for the parameters of these distributions: $[a, b]$ being $[0,1],[0,5]$ and $[-5,10]$; and for the parameters of the Beta distribution the sets $(1,3),(3,1)$ and $(0.5,0.5)$. In the latter case the marginal density is symmetric, as is also the case for the uniform densities.

Frank copula with $\theta=-0.5$

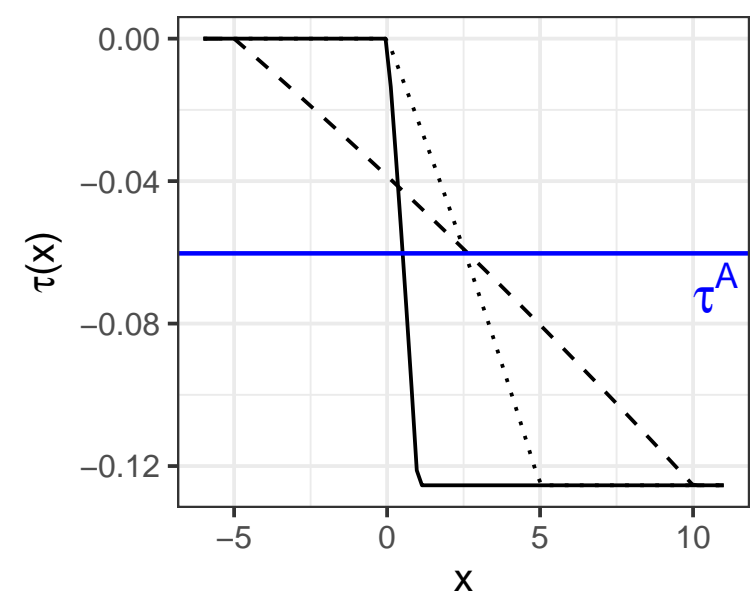

Frank copula with $\theta=3$

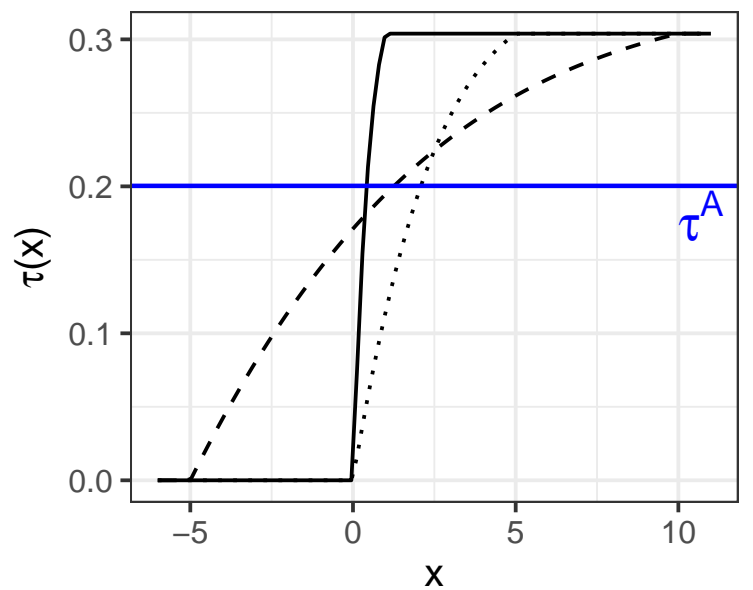

$\mathrm{U}[0,1]$

$\mathrm{U}[0,5]$

$\mathrm{U}[-5,10]$

Figure 4: Frank copula with $\theta=-0.5$ and $\theta=3$. Conditional Kendall's tau for a marginal Uniform distribution for $X$.

Note that the average conditional Kendall's tau is the same for $\theta$ fixed, no matter what the distribution of $X$ is. This is obvious from (20) since the influence of $X$ comes in via the factor $p_{x}(\theta)$, and in that factor it comes in via $F_{X}(x)$ for which of course $F_{X}(X)$ is always uniformly distributed, and hence the average of $\tau(X)$ in (20) only changes with the value of $\theta$. Obviously such a remark is valid in general since from Lemma 4.1 the dependence in $x$ is always via $F_{X}(x)$.

For a Frank copula, the average conditional Kendall's tau and the partial Kendall's tau do not coincide. The integrals in (18) cannot be calculated analytically, but Table 5 reveals that the values of $\tau^{\mathrm{A}}$ and $\bar{\tau}$ are different, but quite close.

Table 5: Frank copula, and values of the average conditional Kendall's tau and partial Kendall's tau.

\begin{tabular}{l|l|l|l}
\hline$\theta$ & 0.5 & 1 & 5 \\
\hline$\tau^{\mathrm{A}}$ & 0.05105 & 0.09368 & 0.24823 \\
$\bar{\tau}$ & 0.05103 & 0.09357 & 0.24707 \\
\hline
\end{tabular}

\section{Bounds for the difference between the partial and average conditional Kendall's tau}

The material in this section complements that of Section 3. In that section the focus was on finding workable conditions under which the partial Kendall's tau and the average conditional Kendall's tau are equal. In this 

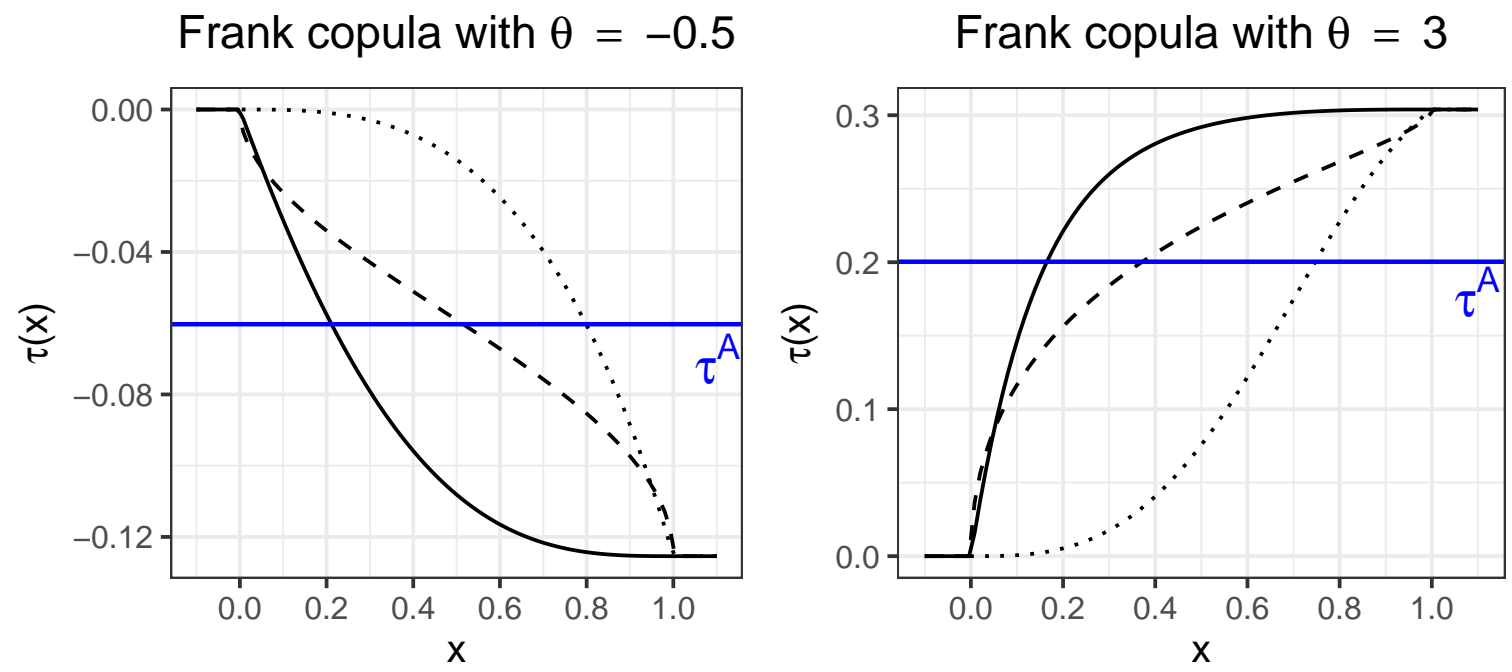

Beta distribution - $\operatorname{Beta}(1,3) \cdots \operatorname{Beta}(3,1) \quad-\quad \operatorname{Beta}(0.5,0.5)$

Figure 5: Frank copula with $\theta=-0.5$ and $\theta=3$. Conditional Kendall's tau for a marginal Beta distribution for $X$.

section we turn attention to trying to provide un upperbound for the difference between $\tau^{\mathrm{A}}$ and $\bar{\tau}$ under Setting 1 in (2).

\subsection{Theoretical results}

A first result can be obtained when $C_{1}$ and $C_{2}$ are ordered copulas. We say that $C_{1}$ is smaller than $C_{2}$, and write

$$
C_{1} \prec C_{2} \quad \text { if } \quad C_{1}\left(u_{1}, u_{2}\right) \leq C_{2}\left(u_{1}, u_{2}\right) \quad \forall\left(u_{1}, u_{2}\right) \in[0,1]^{2} .
$$

Proposition 5.1. Consider $C_{x}$ of the form (2). If $C_{1}$ and $C_{2}$ are such that $C_{1} \prec C_{2}\left(\right.$ or $\left.C_{2} \prec C_{1}\right)$, then $\left|T\left(C_{1}, C_{2}\right)\right| \leq$ $\left|\tau\left(C_{1}\right)-\tau\left(C_{2}\right)\right| / 4 \leq 1 / 2$ and hence

$$
\left|\bar{\tau}-\tau^{A}\right| \leq\left|\tau\left(C_{1}\right)-\tau\left(C_{2}\right)\right| \operatorname{Var}[w(\boldsymbol{X})] \leq 2 \operatorname{Var}[w(\boldsymbol{X})] .
$$

If in addition $C_{1}$ and $C_{2}$ both either describe positive dependence (i.e. $C_{1}, C_{2} \succ \Pi$ ) or negative dependence (i.e. $\left.C_{1}, C_{2} \prec \Pi\right)$, then $\left|T\left(C_{1}, C_{2}\right)\right| \leq 1 / 4$ and hence

$$
\left|\bar{\tau}-\tau^{A}\right| \leq\left|\tau\left(C_{1}\right)-\tau\left(C_{2}\right)\right| \operatorname{Var}[w(\boldsymbol{X})] \leq \operatorname{Var}[w(\boldsymbol{X})] .
$$

Proposition 5.2. Let $C_{\boldsymbol{x}}$ of the form (2). For a mixture of the independence copula $\Pi$ and any other copula $C$, it holds that

and consequently

$$
|T(\Pi, C)|=\frac{|2 \rho(C)-3 \tau(C)|}{12} \leq \frac{1}{12},
$$

$$
\left|\bar{\tau}-\tau^{A}\right| \leq \frac{1}{3} \operatorname{Var}[w(\boldsymbol{X})]
$$

We next consider $C_{1}$ and $C_{2}$ to be members of the same ordered family of copulas. In this case we can establish possibly sharper bounds for $T\left(C_{1}, C_{2}\right)$ in (5) than those in Propositions 5.1 and 5.2. 
More precisely, let $\left\{C_{\theta} \mid \theta \in \Theta\right\}$ be an ordered family of copulas with parameters space $\Theta \subseteq \mathbb{R}$. Denote by

$$
\theta_{\text {inf, } \Theta}=\inf _{\theta}\{\theta \in \Theta\} \quad \text { and } \quad \theta_{\text {sup }, \Theta}=\sup _{\theta}\{\theta \in \Theta\} .
$$

Now consider $C_{1}$ and $C_{2}$ in (2) to come from the same ordered family of copulas $\left\{C_{\theta} \mid \theta \in \Theta\right\}$, i.e. $C_{1}=C_{\theta_{1}}$ and $C_{2}=C_{\theta_{2}}$, with $\theta_{1}, \theta_{2} \in \Theta$. We are interested in evaluating $T\left(C_{1}, C_{2}\right)$. In the sequel we denote

$$
C_{\lim -}\left(u_{1}, u_{2}\right)=C_{\theta \rightarrow \theta_{\text {inf }, \theta}}\left(u_{1}, u_{2}\right) \quad \text { and } \quad C_{\lim +}\left(u_{1}, u_{2}\right)=C_{\theta \rightarrow \theta_{\text {sup }, \theta}}\left(u_{1}, u_{2}\right) .
$$

A copula family is positively (respectively negatively) ordered if

$$
\forall \theta_{1}, \theta_{2} \in \Theta: \theta_{1} \leq \theta_{2} \Longrightarrow \begin{cases}C_{\theta_{1}} \prec C_{\theta_{2}} & \text { in case of a positively ordered family } \\ C_{\theta_{1}} \succ C_{\theta_{2}} & \text { in case of a negatively ordered family. }\end{cases}
$$

If a copula family is positively ordered we thus have that, for every $C$ in that copula family

$$
C_{\lim -} \prec C \prec C_{\lim +},
$$

whereas for a negatively ordered family this statement should be replaced by

$$
C_{\lim -} \succ C \succ C_{\lim +} \cdot
$$

Before stating our next result, we need the following definition. A function $k: \mathbb{R}^{2} \rightarrow \mathbb{R}$ is supermodular on $B \subset \mathbb{R}^{2}$ if

$$
\begin{array}{r}
k\left(y_{1}, y_{2}\right)-k\left(\min \left(x_{1}, y_{1}\right), \min \left(x_{2}, y_{2}\right)\right) \leq k\left(\max \left(x_{1}, y_{1}\right), \max \left(x_{2}, y_{2}\right)\right)-k\left(x_{1}, x_{2}\right) \\
\forall\left(x_{1}, x_{2}\right),\left(y_{1}, y_{2}\right) \in B .
\end{array}
$$

If the function $-k$ is supermodular, then $k$ is called submodular. See for example [26].

Of importance in the sequel is to have some tool to check whether a function possesses the property of being supermodular or submodular on a square $B^{2}$. Some equivalent statements are available via the characterizations of Topkis [34].

\section{Remark 5.1.}

If a function $k$ is twice continuously differentiable, then supermodularity of $k$ on $B^{2}$ is equivalent to checking the condition that

$$
\frac{\partial^{2}}{\partial \theta_{1} \partial \theta_{2}} k\left(\theta_{1}, \theta_{2}\right) \geq 0 \quad \forall\left(\theta_{1}, \theta_{2}\right) \in B
$$

Similarly, $k$ is submodular if and if only all second order mixed partial derivatives are non-positive. This is known as Topkis's characterization theorem. See [34] and [26], among others.

We now define the function

$$
k: \Theta^{2} \rightarrow[0,1 / 2]:\left(\theta_{1}, \theta_{2}\right) \mapsto \iint C_{1} d C_{2} .
$$

Then $T\left(C_{1}, C_{2}\right)$ in (5) can be written as $T\left(C_{1}, C_{2}\right)=-k\left(\theta_{1}, \theta_{1}\right)+k\left(\theta_{2}, \theta_{1}\right)+k\left(\theta_{1}, \theta_{2}\right)-k\left(\theta_{2}, \theta_{2}\right)$.

Proposition 5.3. Let $C_{x}$ be of the form (2).

(1) Assume $C_{1}$ and $C_{2}$ come from the same positively ordered family of copulas $\left\{C_{\theta} \mid \theta \in \Theta\right\}$. Then it holds that

(a) If the function $k$ in (25) is supermodular on $B^{2}=\left[\theta_{1}^{\prime}, \theta_{2}^{\prime}\right]^{2} \subseteq \Theta^{2}$ (with $\theta_{j}^{\prime} \in \Theta$, for $j=1$ ) then $T\left(C_{1}, C_{2}\right) \leq$ 0 .

(b) If the function $k$ in (25) is submodular on $B^{2}=\left[\theta_{1}^{\prime}, \theta_{2}^{\prime}\right]^{2} \subseteq \Theta^{2}$, then $T\left(C_{1}, C_{2}\right) \geq 0$. 
In addition it holds that

$$
\left|T\left(C_{1}, C_{2}\right)\right| \leq\left|T\left(C_{\lim -}, C_{\lim +}\right)\right|,
$$

with the limiting copulas as defined via (22) with $B$ as the parameter space.

(2) Assume that $C_{1}$ and $C_{2}$ are members of the same negatively ordered family of copulas $\left\{C_{\theta} \mid \theta \in \Theta\right\}$. Then (a) and (b) hold, as well as (26) with the limiting copulas as in (22) with B as the parameter space.

One might wonder whether one can give general results about bounding $T\left(C_{1}, C_{2}\right)$ when $C_{1}$ and $C_{2}$ are members of the same copula family, such as members of the same Archimedean family of copulas, or the same class of extreme-value copulas, etc. Achieving general results of this type seems to be quite difficult, but some partial findings can be found in Section S.3 in the Supplementary Material.

\subsection{Examples}

We now illustrate Proposition 5.3 with some examples. We search to compare values of $T\left(C_{1}, C_{2}\right)$ with $T\left(C_{\text {lim }-}, C_{\text {lim }+}\right)$ or $T\left(C_{\lim +}, C_{\text {lim- }}\right)$. For calculation of the integral $\iint_{[0,1]^{2}} C_{1} d C_{2}$ in $T\left(C_{1}, C_{2}\right)$ the following result established in [28, Theorem 5.1.5, page 164] is quite helpful.

Lemma 5.1 ([28]). Let $C_{1}$ and $C_{2}$ be a copulas. Then

$$
\iint_{[0,1]^{2}} C_{1}\left(u_{1}, u_{2}\right) d C_{2}\left(u_{1}, u_{2}\right)=\frac{1}{2}-\iint_{[0,1]^{2}} \frac{\partial}{\partial u_{1}} C_{1}\left(u_{1}, u_{2}\right) \frac{\partial}{\partial u_{2}} C_{2}\left(u_{1}, u_{2}\right) d u_{1} d u_{2}
$$

Example 5.1 (Gumbel-Barnett family).

A copula of the Gumbel-Barnett family has the form

$$
C\left(u_{1}, u_{2}\right)=u_{1} u_{2} \exp \left(-\theta \ln \left(u_{1}\right) \ln \left(u_{2}\right)\right) \quad \theta \in(0,1) .
$$

For $\theta \rightarrow 0$ one obtains the independence copula, and for $\theta \rightarrow 1$ the $\operatorname{copula} C\left(u_{1}, u_{2}\right)=$ $u_{1} u_{2} \exp \left(-\ln \left(u_{1}\right) \ln \left(u_{2}\right)\right)$. We will denote these limiting copulas as $C_{\lim -}$ and $C_{\lim +}$ in the sequel of this example.

This family of copulas is negatively ordered. We use Lemma 5.1 to calculate $\iint_{[0,1]^{2}} C_{1} d C_{2}$, where $C_{j}$ is a Gumbel-Barnett copula with parameter $\theta_{j}$, for $j=1,2$. For the first order partial derivatives of a GumbelBarnett copula $C$ with parameter $\theta$ we obtain

$$
\begin{aligned}
& \frac{\partial}{\partial u_{1}} C\left(u_{1}, u_{2}\right)=\left(u_{2}-\theta u_{2} \ln u_{2}\right) \exp \left(-\theta \ln u_{1} \ln u_{2}\right) \\
& \frac{\partial}{\partial u_{2}} C\left(u_{1}, u_{2}\right)=\left(u_{1}-\theta u_{1} \ln u_{1}\right) \exp \left(-\theta \ln u_{1} \ln u_{2}\right)
\end{aligned}
$$


Using Lemma 5.1 we get

$$
\begin{aligned}
& \iint_{[0,1]^{2}} C_{1}\left(u_{1}, u_{2}\right) d C_{2}\left(u_{1}, u_{2}\right) \\
& =\frac{1}{2}-\iint\left(u_{2}-\theta_{1} u_{2} \ln u_{2}\right)\left(u_{1}-\theta_{2} u_{1} \ln u_{1}\right) \exp \left(-\left(\theta_{1}+\theta_{2}\right) \ln u_{1} \ln u_{2}\right) d u_{1} d u_{2} \\
& =\frac{1}{2}-\iint u_{1} u_{2} \exp \left(-\left(\theta_{1}+\theta_{2}\right) \ln u_{1} \ln u_{2}\right) d u_{1} d u_{2} \\
& \quad+\theta_{1} \iint u_{1} u_{2} \ln u_{2} \exp \left(-\left(\theta_{1}+\theta_{2}\right) \ln u_{1} \ln u_{2}\right) d u_{1} d u_{2} \\
& \quad+\theta_{2} \iint u_{1} u_{2} \ln u_{1} \exp \left(-\left(\theta_{1}+\theta_{2}\right) \ln u_{1} \ln u_{2}\right) d u_{1} d u_{2} \\
& \quad-\theta_{1} \theta_{2} \iint u_{1} u_{2} \ln u_{1} \ln u_{2} \exp \left(-\left(\theta_{1}+\theta_{2}\right) \ln u_{1} \ln u_{2}\right) d u_{1} d u_{2} \\
& \equiv \frac{1}{2}+T_{1}+T_{2}+T_{3}+T_{4} .
\end{aligned}
$$

Details of the straightforward but a bit tedious calculation of the four integrals are provided in Section S.2 of the Supplementary Material and give that

$$
\begin{aligned}
\iint_{[0,1]^{2}} C_{1}\left(u_{1}, u_{2}\right) d C_{2}\left(u_{1}, u_{2}\right)= & \frac{-\theta_{1}^{2}-\theta_{2}^{2}+2 \theta_{1} \theta_{2}+\theta_{1} \theta_{2}\left(\theta_{1}+\theta_{2}\right)}{\left(\theta_{1}+\theta_{2}\right)^{3}} e^{\frac{4}{\theta_{1}+\theta_{2}}} \mathrm{Ei}\left(-\frac{4}{\theta_{1}+\theta_{2}}\right) \\
& +\frac{\theta_{1} \theta_{2}}{\left(\theta_{1}+\theta_{2}\right)^{2}},
\end{aligned}
$$

where $\operatorname{Ei}(x)=-\int_{-x}^{\infty} \frac{e^{-t}}{t} d t$ is the exponential integral function. Obviously, when $\theta_{1}=\theta_{2}$, this simplifies to

$$
\iint_{[0,1]^{2}} C_{1}\left(u_{1}, u_{2}\right) d C_{1}\left(u_{1}, u_{2}\right)=\frac{1}{4}\left(e^{\frac{2}{\theta_{1}}} \operatorname{Ei}\left(\frac{-2}{\theta_{1}}\right)+1\right),
$$

and similarly for $\iint C_{2} d C_{2}$, replacing $\theta_{1}$ with $\theta_{2}$ in the above expression.

Furthermore, one can show that the function $k\left(\theta_{1}, \theta_{2}\right):=\iint_{[0,1]^{2}} C_{1} d C_{2}$ is submodular on $(0,1)^{2}$, which is the entire domain of the copula. Indeed, since

$$
\begin{aligned}
& \frac{\partial^{2} k}{\partial \theta_{1} \partial \theta_{2}}\left(\theta_{1}, \theta_{2}\right) \\
& \begin{array}{l}
=\frac{1}{\left(\theta_{1}+\theta_{2}\right)^{7}}\left\{-\left[\theta_{2}^{5}+\left(-\theta_{1}+14\right) \theta_{2}^{4}+\left(-8 \theta_{1}^{2}-16 \theta_{1}+32\right) \theta_{2}^{3}\right.\right. \\
\left.\quad+\left(-8 \theta_{1}^{3}-60 \theta_{1}^{2}-48 \theta_{1}+16\right) \theta_{2}^{2}-\left(\theta_{1}^{4}+16 \theta_{1}^{3}+48 \theta_{1}^{2}+32 \theta_{1}\right) \theta_{2}+\theta_{1}^{2}\left(\theta_{1}+2\right)\left(\theta_{1}^{2}+12 \theta_{1}+8\right)\right] \\
\quad \times e^{\frac{4}{\theta_{1}+\theta_{2}}} \mathrm{Ei}\left(\frac{-4}{\theta_{1}+\theta_{2}}\right)
\end{array} \\
& \begin{array}{l}
-2\left(\theta_{1}+\theta_{2}\right) \quad \\
\left.\quad\left[\theta_{2}^{4}+\left(-\frac{3}{2} \theta_{1}+\frac{7}{2}\right) \theta_{2}^{3}+\left(-5 \theta_{1}^{2}-\frac{11}{2} \theta_{1}+2\right) \theta_{2}^{2}-\left(\frac{3}{2} \theta_{1}^{3}+\frac{11}{2} \theta_{1}^{2}+4 \theta_{1}\right) \theta_{2}+\theta_{1}^{2}\left(\theta_{1}^{2}+\frac{7}{2} \theta_{1}+2\right)\right]\right\},
\end{array}
\end{aligned}
$$

which is negative for all $\left(\theta_{1}, \theta_{2}\right) \in(0,1)^{2}$ it follows from (24) and Remark 5.1 that $k$ is submodular. From Proposition 5.3 we thus know that

$$
0 \leq T\left(C_{1}, C_{2}\right) \leq T\left(C_{\text {lim- }}, C_{\text {lim+ }}\right) .
$$

For the lower and upper copula limits, $C_{\text {lim - }}$ and $C_{\text {lim + }}$, we find from (28) and (29) that

$$
\begin{aligned}
& \iint_{[0,1]^{2}} C_{\lim -} d C_{\lim -}=\frac{1}{4}, \quad \iint_{[0,1]^{2}} C_{\lim -} d C_{\lim +}=-e^{4} \operatorname{Ei}(-4) \\
& \text { and } \iint_{\mathbf{I}^{2}} C_{\lim +} d C_{\lim +}=\frac{1}{4}\left(e^{2} \operatorname{Ei}(-2)+1\right),
\end{aligned}
$$


where $\operatorname{Ei}(x)$ can be calculated numerically. Consequently we find

$$
\begin{aligned}
T\left(C_{\lim -}, C_{\lim +}\right) & =-\iint_{[0,1]^{2}} C_{\lim -} d C_{\lim -}+2 \iint_{\mathbf{I}^{2}} C_{\lim -} d C_{\lim +}-\iint_{[0,1]^{2}} C_{\lim +} d C_{\lim +} \\
& =-\frac{1}{4}+2\left[-e^{4} \operatorname{Ei}(-4)\right]-\frac{1}{4}\left(e^{2} \operatorname{Ei}(-2)+1\right) \\
& =-\frac{1}{2}+\frac{e^{2}}{4} \operatorname{Ei}(-2)-2 e^{4} \operatorname{Ei}(-4) \\
& \approx 0.00302 .
\end{aligned}
$$

Figure 6 presents $T\left(C_{1}, C_{2}\right)=\left|T\left(C_{1}, C_{2}\right)\right|$ over the whole range of parameter values $\left(\theta_{1}, \theta_{2}\right)$.

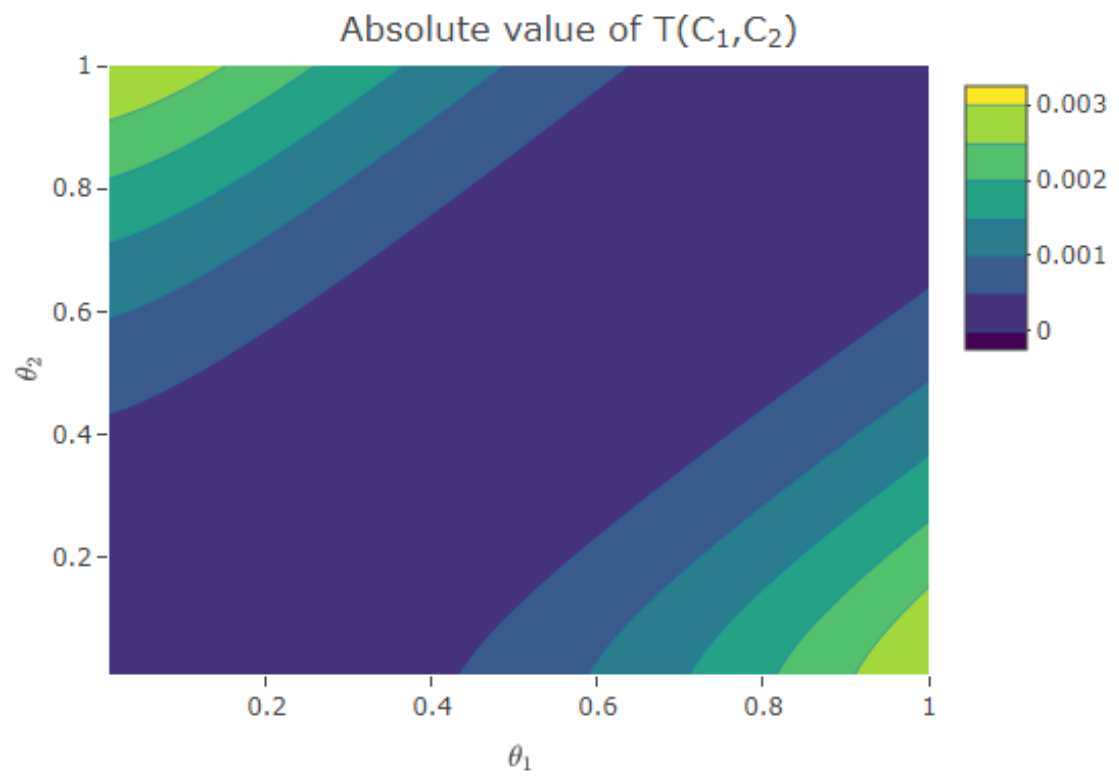

Figure 6: $\left|T\left(C_{1}, C_{2}\right)\right|$ in function of the parameters $\theta_{1}$ and $\theta_{2}$, for two Gumbel-Barnett copulas $C_{1}$ and $C_{2}$.

Example 5.2 (Ali-Mikhail-Haq family).

An Ali-Mikhail-Haq copula takes the form

$$
C\left(u_{1}, u_{2}\right)=\frac{u_{1} u_{2}}{1-\theta\left(1-u_{1}\right)\left(1-u_{2}\right)} \quad \theta \in[-1,1) .
$$

The Ali-Mikhail-Haq family of copulas is positively ordered, and describes both positive and negative dependencies. For $\theta=0$, we get the independence copula. The first order partial derivatives of an Ali-Mikhail-Haq copula $C$ with parameter $\theta$ are

$$
\frac{\partial}{\partial u_{1}} C\left(u_{1}, u_{2}\right)=\frac{\left[1-\theta\left(1-u_{2}\right)\right] u_{2}}{\left[1-\theta\left(1-u_{1}\right)\left(1-u_{2}\right)\right]^{2}} \quad \text { and } \quad \frac{\partial}{\partial u_{2}} C\left(u_{1}, u_{2}\right)=\frac{\left[1-\theta\left(1-u_{1}\right)\right] u_{1}}{\left[1-\theta\left(1-u_{1}\right)\left(1-u_{2}\right)\right]^{2}} .
$$


Consider now two Ali-Mikhail-Haq copulas, $C_{1}$ and $C_{2}$, with respective parameters $\theta_{1}$ and $\theta_{2}$. Using Lemma 5.1 we get after some calculations (see Section S.2 of the Supplementary Material) that

$$
\begin{aligned}
& \iint_{[0,1]^{2}} C_{1}\left(u_{1}, u_{2}\right) d C_{2}\left(u_{1}, u_{2}\right) \\
&=\frac{1}{2}-\frac{1}{\left(\theta_{2}-\theta_{1}\right)^{3}}[-\left(\theta_{1} \theta_{2}^{2}+\theta_{1}^{2} \theta_{2}+\theta_{2}^{2}+6 \theta_{1} \theta_{2}+\theta_{1}^{2}+\theta_{2}+\theta_{1}\right)\left[\operatorname{dilog}\left(\theta_{2}\right)-\operatorname{dilog}\left(\theta_{1}\right)\right] \\
&+2\left(1-\theta_{2}\right)\left(2 \theta_{1} \theta_{2}+\theta_{2}+\theta_{1}^{2}+2 \theta_{1}\right) \ln \left(1-\theta_{2}\right) \\
&-2\left(1-\theta_{1}\right)\left(\theta_{2}^{2}+2 \theta_{1} \theta_{2}+2 \theta_{2}+\theta_{1}\right) \ln \left(1-\theta_{1}\right) \\
&\left.+\frac{\theta_{2}-\theta_{1}}{2}\left(\theta_{2}^{2}+10 \theta_{1} \theta_{2}+6 \theta_{2}+\theta_{1}^{2}+6 \theta_{1}\right)\right],
\end{aligned}
$$

where dilog $(x)=\int_{x}^{0} \frac{\ln (1-t)}{t} d t$ is the so-called dilogarithm function, also known as Spence's function.

One can check that the limit of (31) when $\theta_{2} \rightarrow \theta_{1}$ leads to the following expression

$$
\iint_{[0,1]^{2}} C_{1}\left(u_{1}, u_{2}\right) d C_{1}\left(u_{1}, u_{2}\right)=\frac{1}{2}-\frac{1}{6} \frac{\ln \left(1-\theta_{1}\right)\left(1-\theta_{1}\right)^{2}+\theta_{1}}{\theta_{1}^{2}} .
$$

In the light of Proposition 5.3 we check the sign of the second order mixed partial derivatives of the function $k\left(\theta_{1}, \theta_{2}\right):=\iint_{[0,1]^{2}} C_{1} d C_{2}$. The expression of this derivative is

$$
\begin{aligned}
& \frac{\partial^{2} k}{\partial \theta_{1} \partial \theta_{2}}\left(\theta_{1}, \theta_{2}\right) \\
& =\frac{1}{\theta_{1} \theta_{2}\left(\theta_{2}-\theta_{1}\right)^{5}}\left[\theta_{2}\left(\theta_{1}-1\right)\left[\theta_{2}{ }^{3}+\left(27 \theta_{1}+2\right) \theta_{2}{ }^{2}+\left(39 \theta_{1}{ }^{2}+44 \theta_{1}\right) \theta_{2}+\theta_{1}^{2}\left(5 \theta_{1}+26\right)\right] \ln \left(1-\theta_{1}\right)\right. \\
& -\theta_{1}\left(\theta_{2}-1\right)\left(5 \theta_{2}{ }^{3}+\left(39 \theta_{1}+26\right) \theta_{2}{ }^{2}+\left(27 \theta_{1}{ }^{2}+44 \theta_{1}\right) \theta_{2}+\theta_{1}{ }^{2}\left(\theta_{1}+2\right)\right) \ln \left(1-\theta_{2}\right) \\
& +\theta_{1} \theta_{2}\left[\theta_{2}^{3}+\left(11 \theta_{1}+18\right) \theta_{2}^{2}+\left(11 \theta_{1}^{2}+60 \theta_{1}+12\right) \theta_{2}+\theta_{1}^{3}+18 \theta_{1}^{2}+12 \theta_{1}\right] \\
& \times\left[\operatorname{dil} \log \left(\theta_{1}\right)-\operatorname{dilog}\left(\theta_{2}\right)\right] \\
& \left.+12 \theta_{1} \theta_{2}\left(\theta_{2}-\theta_{1}\right)\left[\theta_{2}^{2}+\left(4 \theta_{1}+3\right) \theta_{2}+\theta_{1}^{2}+3 \theta_{1}\right]\right],
\end{aligned}
$$

which in the limit when $\theta_{2} \rightarrow \theta_{1}$, results into

$$
\lim _{\theta_{2} \rightarrow \theta_{1}} \frac{\partial^{2}}{\partial \theta_{1} \partial \theta_{2}}\left(\iint_{0,1]^{2}} C_{1}\left(u_{1}, u_{2}\right) d C_{2}\left(u_{1}, u_{2}\right)\right)=\frac{-\ln \left(1-\theta_{1}\right)\left(\theta_{1}^{2}-6 \theta_{1}+6\right)+3 \theta_{1}\left(\theta_{1}-2\right)}{30 \theta_{1}^{4}} .
$$

The limit of this expression when $\theta_{1}$ tends to 0 is zero. From (33) it is clear that, for all $\left(\theta_{1}, \theta_{2}\right)$, the second order partial derivative is non-positive on a subset $A$ of $[-1,1)^{2}$ and non-negative on $A^{C}=[-1,1)^{2} \backslash A$. Figure 7 depicts the function $\frac{\partial^{2} k}{\partial \theta_{1} \partial \theta_{2}}$. On the darkest part (the set $A$ ), $\frac{\partial^{2} k}{\partial \theta_{1} \partial \theta_{2}} \leq 0$ and so $k$ is submodular. On the other part (the set $A^{C}$ ) this mixed derivative of $k$ is non-negative, and therefore $k$ is supermodular.

Since, for example, $k$ is submodular on $B^{2}=[-1,0]^{2}$, and $k$ is supermodular on $B^{2}=[0,1)^{2}$, we can apply Proposition 5.3. First consider the interval $B=[-1,0]$, with $\theta_{\text {inf, } B}=\inf _{\theta}\{\theta \in \Theta\}=-1$ and $\theta_{\text {sup }, B}=$ $\sup _{\theta}\{\theta \in \Theta\}=0$. From (32) and (31), we find

$$
\begin{aligned}
& \iint_{[0,1]^{2}} C_{\lim -} d C_{\lim -}=\frac{2}{3}[1-\ln (2)], \quad \iint_{[0,1]^{2}} C_{\lim +} d C_{\lim +}=\frac{1}{4}, \\
& \text { and } \iint_{[0,1]^{2}} C_{\lim -} d C_{\lim +}=3-4 \ln (2)
\end{aligned}
$$




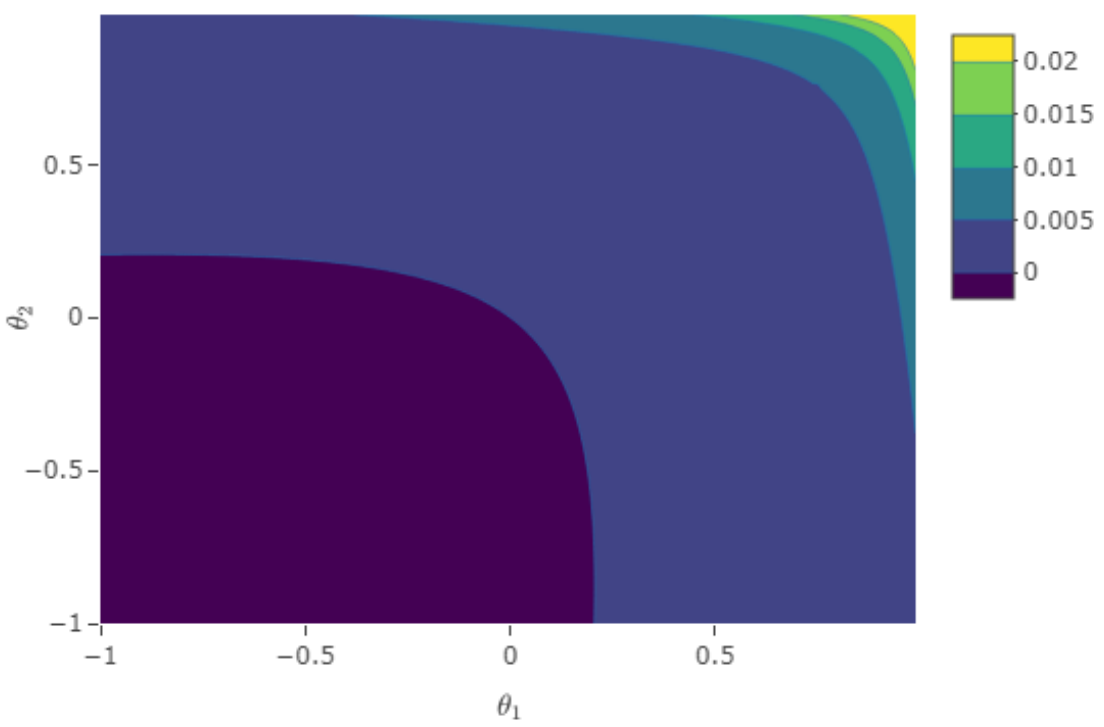

Figure 7: Ali-Mikhail-Haq copula. The second order mixed partial derivative function $\frac{\partial^{2} k}{\partial \theta_{1} \partial \theta_{2}}$.

and hence

$$
\begin{aligned}
T\left(C_{\lim -}, C_{\lim +}\right) & =-\iint_{[0,1]^{2}} C_{\lim -} d C_{\lim -}+2 \iint_{[0,1]^{2}} C_{\lim -} d C_{\lim +}-\iint_{[0,1]^{2}} C_{\lim +} d C_{\lim +} \\
& =-\frac{2}{3}[1-\ln (2)]+2[3-4 \ln (2)]-\frac{1}{4} \\
& =\frac{61}{12}-\frac{22}{3} \ln (2) \\
& \approx 0.000254
\end{aligned}
$$

and consequently, by applying Proposition 5.3

$$
0 \leq T\left(C_{1}, C_{2}\right) \leq T\left(C_{\theta_{1}=-1}, C_{\theta_{2}=0}\right)=0.000254 \quad \text { on }[-1,0] .
$$

Similarly, considering $B=[0,1)$, with $\theta_{\text {inf }, B}=\inf _{\theta}\{\theta \in \Theta\}=0$ and $\theta_{\text {sup }, B}=\sup _{\theta}\{\theta \in \Theta\}=1$, and obtaining from (32) and (31) that

$$
\iint_{[0,1]^{2}} C_{\lim -} d C_{\lim +}=-3+2 \operatorname{dilog}(1),
$$

and

$$
\iint_{[0,1]^{2}} C_{\lim -} d C_{\lim -}=\frac{1}{4}, \quad \iint_{[0,1]^{2}} C_{\lim +} d C_{\lim +}=\frac{1}{3},
$$

we get

$$
\begin{aligned}
T\left(C_{\lim -}, C_{\lim +}\right) & =-\iint_{[0,1]^{2}} C_{\lim -} d C_{\lim -}+2 \iint_{[0,1]^{2}} C_{\lim -} d C_{\lim +}-\iint_{[0,1]^{2}} C_{\lim +} d C_{\lim +} \\
& =-\frac{1}{4}++2[-3+2 \operatorname{dilog}(1)]-\frac{1}{3} \\
& =-\frac{7}{12}-6+4 \operatorname{dilog}(1) \\
& \approx-0.00360 .
\end{aligned}
$$




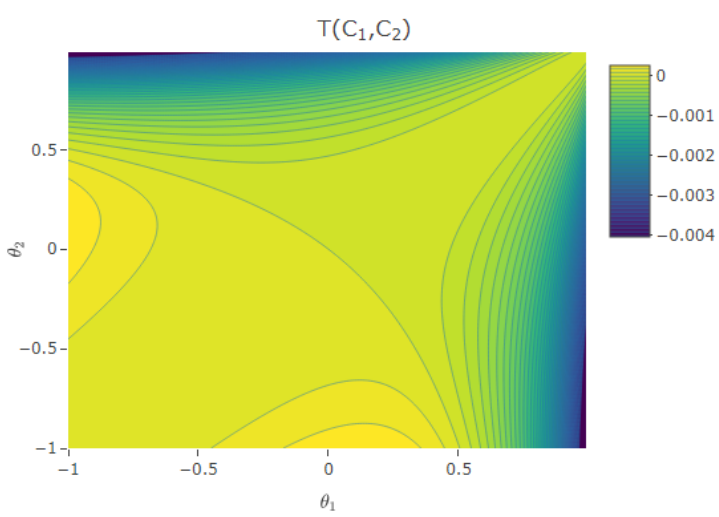

(a)

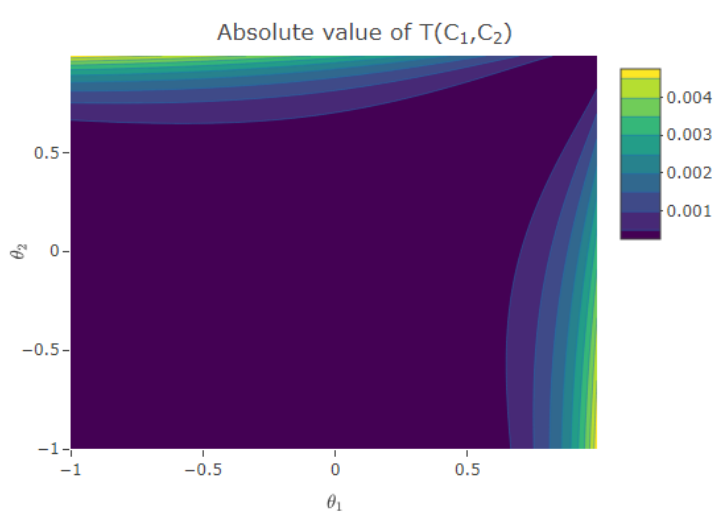

(b)

Figure 8: $T\left(C_{1}, C_{2}\right)$ and $\left|T\left(C_{1}, C_{2}\right)\right|$ in function of the parameters $\theta_{1}$ and $\theta_{2}$ of two AMH copulas $C_{1}$ and $C_{2}$.

From Proposition 5.3 we then get

$$
0 \geq T\left(C_{1}, C_{2}\right) \geq T\left(C_{\theta_{1}=0}, C_{\theta_{2} \rightarrow 1}\right)=-0.00360 \quad \text { on }[0,1)
$$

Figure 8 (left) shows how $T\left(C_{1}, C_{2}\right)$ differs for different parameter values of the two AMH copulas, whereas $\left|T\left(C_{1}, C_{2}\right)\right|$ is shown on the right-hand side of the figure. Note that $T\left(C_{1}, C_{2}\right)$ takes on positive values on $[-1,0]^{2}$, and negative values on, for example, $[0,1)^{2}$.

\section{Ordering between partial and average conditional Kendall's tau}

Consider couples of random variables $\boldsymbol{Y}=\left(Y_{1}, Y_{2}\right)^{\mathrm{T}}$ and $\boldsymbol{Z}=\left(Z_{1}, Z_{2}\right)^{\mathrm{T}}$. The interest is in the conditional dependencies between the components of $\boldsymbol{Y}$ given $\boldsymbol{X}$, and similarly these between the components of $\boldsymbol{Z}$ given $\boldsymbol{X}$. Denote the average conditional Kendall's tau's for $\boldsymbol{Y}$ and $\boldsymbol{Z}$ by $\tau_{\boldsymbol{Y}}^{\mathrm{A}}$ and $\tau_{\boldsymbol{Z}}^{\mathrm{A}}$ respectively. Similarly, the partial Kendall's tau's for both couples are denoted by $\bar{\tau}_{\boldsymbol{Y}}$ and $\bar{\tau}_{\boldsymbol{Z}}$ respectively. An interesting question is whether $\tau_{\boldsymbol{Y}}^{\mathrm{A}} \leq \tau_{\boldsymbol{Z}}^{\mathrm{A}}$ implies that $\bar{\tau}_{\boldsymbol{Y}} \leq \bar{\tau}_{\boldsymbol{Z}}$, or vice versa. As will be seen from the sequel, such an implication or equivalence does not hold in general.

To investigate the above question, we focus for simplicity on Setting 1, and consider the conditional copulas of $\boldsymbol{Y}$ given $\boldsymbol{X}=\boldsymbol{x}$, and of $\boldsymbol{Z}$ given $\boldsymbol{X}=\boldsymbol{x}$ to be of the form

$$
\begin{aligned}
& C_{\boldsymbol{Y} \mid \boldsymbol{X}=\boldsymbol{x}}\left(u_{1}, u_{2}\right)=w(\boldsymbol{x}) C_{1}\left(u_{1}, u_{2}\right)+(1-w(\boldsymbol{x})) C_{2}\left(u_{1}, u_{2}\right) \\
& C_{\boldsymbol{Z} \mid \boldsymbol{X}=\boldsymbol{x}}\left(u_{1}, u_{2}\right)=w(\boldsymbol{x}) C_{3}\left(u_{1}, u_{2}\right)+(1-w(\boldsymbol{x})) C_{4}\left(u_{1}, u_{2}\right),
\end{aligned}
$$

for copulas $C_{1}, C_{2}, C_{3}$ and $C_{4}$. For simplicity of presentation we introduce some notations

$$
\begin{aligned}
D & =\left(\iint C_{3} d C_{4}-\iint C_{1} d C_{2}\right)^{2}-\left(\iint C_{3} d C_{3}-\iint C_{1} d C_{1}\right)\left(\iint C_{4} d C_{4}-\iint C_{2} d C_{2}\right) \\
b & =\iint C_{1} d C_{2}-\iint C_{2} d C_{2}-\iint C_{3} d C_{4}+\iint C_{4} d C_{4} .
\end{aligned}
$$

Recall also the notation $\mu_{\ell}=\mathrm{E}_{\boldsymbol{X}}\left[w^{\ell}(\boldsymbol{X})\right]$, for $\ell=1,2$.

Theorem 6.1. Suppose that the dependence structures of $\boldsymbol{Y} \mid \boldsymbol{X}=\boldsymbol{x}$ and $\boldsymbol{Z} \mid \boldsymbol{X}=\boldsymbol{x}$ are respectively given by the conditional mixture copulas (34). 
(i). If $T\left(C_{1}, C_{2}\right)>T\left(C_{3}, C_{4}\right)$, then

$$
\tau_{\boldsymbol{Y}}^{A} \leq \tau_{\boldsymbol{Z}}^{A} \Longleftrightarrow \mu_{2} \geq \frac{2 \mu_{1} b-\iint C_{4} d C_{4}+\iint C_{2} d C_{2}}{T\left(C_{1}, C_{2}\right)-T\left(C_{3}, C_{4}\right)}
$$

and

(a) if $D>0$, then

$$
\bar{\tau}_{Y}>\bar{\tau}_{Z} \Longleftrightarrow \frac{b-\sqrt{D}}{T\left(C_{1}, C_{2}\right)-T\left(C_{3}, C_{4}\right)}<\mu_{1}<\frac{b+\sqrt{D}}{T\left(C_{1}, C_{2}\right)-T\left(C_{3}, C_{4}\right)} ;
$$

(b) if $D \leq 0$, then $\bar{\tau}_{\boldsymbol{Y}} \leq \bar{\tau}_{\boldsymbol{Z}}$ for all $\mu_{1} \in[0,1]$.

(ii). If $T\left(C_{1}, C_{2}\right)<T\left(C_{3}, C_{4}\right)$, then

$$
\tau_{\boldsymbol{Y}}^{A} \leq \tau_{\boldsymbol{Z}}^{A} \Longleftrightarrow \mu_{2} \leq \frac{2 \mu_{1} b-\iint C_{4} d C_{4}+\iint C_{2} d C_{2}}{T\left(C_{1}, C_{2}\right)-T\left(C_{3}, C_{4}\right)}
$$

and

(a) if $D>0$, then

$$
\bar{\tau}_{\boldsymbol{Y}}>\bar{\tau}_{\boldsymbol{Z}} \Longleftrightarrow \mu_{1}<\frac{b+\sqrt{D}}{T\left(C_{1}, C_{2}\right)-T\left(C_{3}, C_{4}\right)} \quad \text { and } \quad \mu_{1}>\frac{b-\sqrt{D}}{T\left(C_{1}, C_{2}\right)-T\left(C_{3}, C_{4}\right)} ;
$$

(b) if $D=0$, then

$$
\bar{\tau}_{\boldsymbol{Y}}>\bar{\tau}_{\boldsymbol{Z}} \Longleftrightarrow \mu_{1} \neq \frac{b}{T\left(C_{1}, C_{2}\right)-T\left(C_{3}, C_{4}\right)} ;
$$

(c) if $D<0$, then $\bar{\tau}_{\boldsymbol{Y}}>\bar{\tau}_{Z}$ for all $\mu_{1} \in[0,1]$.

(iii). If $T\left(C_{1}, C_{2}\right)=T\left(C_{3}, C_{4}\right)$, then $\tau_{\boldsymbol{Y}}^{A} \leq \tau_{Z}^{A} \Longleftrightarrow \bar{\tau}_{\boldsymbol{Y}} \leq \bar{\tau}_{\boldsymbol{Z}}$.

Moreover, if $C_{3} \prec C_{1}$ and $C_{2} \prec C_{4}$ (or $C_{1} \prec C_{3}$ and $C_{4} \prec C_{2}$ ), then $D \geq 0$.

Given four copulas, Theorem 6.1 thus provides conditions on $\mu_{1}$ and $\mu_{2}$ such that $\tau_{Y}^{\mathrm{A}} \leq \tau_{Z}^{\mathrm{A}}$ and $\bar{\tau}_{\boldsymbol{Y}}>\bar{\tau}_{Z}$. So any distribution (for $w(\boldsymbol{X})$ ) on $[0,1]$ that satisfies the above conditions, will result in a counterexample for the statement that $\tau_{Y}^{\mathrm{A}} \leq \tau_{Z}^{\mathrm{A}}$ implies $\bar{\tau}_{\boldsymbol{Y}} \leq \bar{\tau}_{Z}$, or vice versa. We provide some examples to illustrate these findings.

The first example shows that $\tau_{\boldsymbol{Y}}^{\mathrm{A}} \leq \tau_{\boldsymbol{Z}}^{\mathrm{A}}$ does not necessarily imply that also $\bar{\tau}_{\boldsymbol{Y}} \leq \bar{\tau}_{\boldsymbol{Z}}$.

\section{Example 6.1.}

Suppose that the dependence structures of $\boldsymbol{Y}$ given $\boldsymbol{X}$ and $\boldsymbol{Z}$ given $\boldsymbol{X}$ are given by (34) with $C_{1}=C_{4}=M$, $C_{2}=W$ and $C_{3}=\Pi$. Since $\Pi \prec M$ and $W \prec M$, we know from Theorem 6.1 that $D \geq 0$, namely here $D=19 / 144$. We also have that $T\left(C_{1}, C_{2}\right)-T\left(C_{3}, C_{4}\right)=1 / 12$. So we find that

$$
\tau_{\boldsymbol{Y}}^{\mathrm{A}} \leq \tau_{\boldsymbol{Z}}^{\mathrm{A}} \Longleftrightarrow \mu_{2} \geq 10 \mu_{1}-6
$$

and

$$
\bar{\tau}_{Y}>\bar{\tau}_{Z} \Longleftrightarrow 5-\sqrt{19}<\mu_{1}<5+\sqrt{19} .
$$

Together with the assumption that $0 \leq w(x) \leq 1$, we get that any distribution of $w(X)$ with $5-\sqrt{19}<\mu_{1} \leq 1$ and $\mu_{2} \geq 10 \mu_{1}-6$ results in an example where $\tau_{\boldsymbol{Y}}^{\mathrm{A}} \leq \tau_{\boldsymbol{Z}}^{\mathrm{A}}$ but not $\bar{\tau}_{\boldsymbol{Y}} \leq \bar{\tau}_{\boldsymbol{Z}}$.

For example, if $w(\boldsymbol{X})$ has a Beta distribution with parameters 5 and 2.77, then $\mu_{1}=0.64350$ and $\mu_{2}=$ 0.44025 , and the above conditions on $\mu_{2}$ and $\mu_{1}$ are fulfilled. In this case, we get the following values for the average conditional Kendall's tau's and the partial Kendall's tau's:

$$
\tau_{\boldsymbol{Y}}^{\mathrm{A}}=0.28700, \quad \tau_{\boldsymbol{Z}}^{\mathrm{A}}=0.28875, \quad \bar{\tau}_{\boldsymbol{Y}}=0.28700 \quad \text { and } \quad \bar{\tau}_{\boldsymbol{Z}}=0.28003 .
$$


So, indeed $\tau_{\boldsymbol{Y}}^{\mathrm{A}} \leq \tau_{\boldsymbol{Z}}^{\mathrm{A}}$ whereas $\bar{\tau}_{\boldsymbol{Y}}>\bar{\tau}_{\boldsymbol{Z}}$.

The next example provides an example in which $D<0$.

\section{Example 6.2.}

Taking $C_{1}=W, C_{2}=\Pi, C_{3}=\Pi$ and $C_{4}=M$ in (34), results in $T\left(C_{1}, C_{2}\right)-T\left(C_{3}, C_{4}\right)=1 / 6$ and

$$
D=\left(\frac{1}{3}-\frac{1}{6}\right)^{2}-\frac{1}{4}\left(\frac{1}{2}-\frac{1}{4}\right)=-5 / 144 \text {. }
$$

Hence, applying Theorem 6.1 we know that

$$
\tau_{\boldsymbol{Y}}^{\mathrm{A}} \leq \tau_{\boldsymbol{Z}}^{\mathrm{A}} \Longleftrightarrow \mu_{2} \geq \mu_{1}-\frac{3}{2},
$$

which is always true for $\mu_{1}, \mu_{2} \in[0,1]$, and

$$
\bar{\tau}_{Y} \leq \bar{\tau}_{Z}
$$

So, in this example we have that $\tau_{\boldsymbol{Y}}^{\mathrm{A}} \leq \tau_{\boldsymbol{Z}}^{\mathrm{A}}$ and $\bar{\tau}_{\boldsymbol{Y}} \leq \bar{\tau}_{\boldsymbol{Z}}$ for all $\mu_{1}$ and $\mu_{2}$.

An answer to our initial question is provided in the following result, that follows from Theorem 6.1.

Corollary 6.1. Suppose that the dependence structures of $\boldsymbol{Y} \mid \boldsymbol{X}=\boldsymbol{x}$ and $\boldsymbol{Z} \mid \boldsymbol{X}=\boldsymbol{x}$ are given by the conditional mixture copulas (34). Then the following statements hold.

(i). If $T\left(C_{1}, C_{2}\right)-T\left(C_{3}, C_{4}\right)>0$, then $\bar{\tau}_{\boldsymbol{Y}} \leq \bar{\tau}_{\boldsymbol{Z}} \Longrightarrow \tau_{\boldsymbol{Y}}^{A} \leq \tau_{\boldsymbol{Z}}^{A}$.

Furthermore, if $D \leq 0$, then not only $\bar{\tau}_{\boldsymbol{Y}} \leq \bar{\tau}_{\boldsymbol{Z}}$ but also $\tau_{\boldsymbol{Y}}^{A} \leq \tau_{\boldsymbol{Z}}^{A}$ holds.

(ii). If $T\left(C_{1}, C_{2}\right)-T\left(C_{3}, C_{4}\right)<0$, then $\bar{\tau}_{\boldsymbol{Y}} \geq \bar{\tau}_{\boldsymbol{Z}} \Rightarrow \tau_{\boldsymbol{Y}}^{A} \geq \tau_{\boldsymbol{Z}}^{A}$.

If additionally $D<0$, then not only $\bar{\tau}_{\boldsymbol{Y}}>\bar{\tau}_{\boldsymbol{Z}}$ but also $\tau_{\boldsymbol{Y}}^{A}>\tau_{\boldsymbol{Z}}^{A}$.

\section{Extension to multivariate conditional dependencies}

So far, the focus was on the conditional dependence between two random variables, say $Y_{1}$ and $Y_{2}$, conditionally upon a covariate $\boldsymbol{X}$ (or $X$ in Setting 2). Many (but not all) of the results established in Sections 3-6 continue to hold in case of a $p$-dimensional vector $\boldsymbol{Y}=\left(Y_{1}, \ldots, Y_{p}\right)^{\mathrm{T}}$ (with $p \geq 2$ ) where the interest is in the conditional dependence structure between the components of $\boldsymbol{Y}$ given a covariate vector $\boldsymbol{X}$. In this section we briefly comment on multivariate generalizations of the results in Sections 3, 4 and 5.

\subsection{Multivariate Kendall's tau}

Consider that the dependence structure of the random variables $Y_{1}, \ldots, Y_{p}$ is described by the multivariate copula $C(\boldsymbol{u})=C\left(u_{1}, u_{2}, \ldots, u_{p}\right)$. A multivariate version of Kendall's tau is given by

$$
\tau_{\boldsymbol{Y}}=\frac{1}{2^{p-1}-1}\left\{2^{p} \int_{[0,1]^{p}} C(\boldsymbol{u}) d C(\boldsymbol{u})-1\right\} .
$$

which we also denote below as $\tau(C)$. See [28]. 
There are several generalizations of Spearman's rho. See [17] for a recent discussion on these. A first generalization proposed by [36] is

$$
\rho_{1, \boldsymbol{Y}}=\frac{p+1}{2^{p}-(p+1)}\left\{2^{p} \int_{[0,1]^{p}} C(\boldsymbol{u}) d \boldsymbol{u}-1\right\} .
$$

Another generalization introduced by [24] is

$$
\rho_{2, \boldsymbol{Y}}=\frac{p+1}{2^{p}-(p+1)}\left\{2^{p} \int_{[0,1]^{p}} \Pi(\boldsymbol{u}) d C(\boldsymbol{u})-1\right\},
$$

with $\Pi(\boldsymbol{u})=\prod_{j=1}^{p} u_{j}$ the $p$-dimensional independence copula. We also use the notations $\rho_{1}(C)$ and $\rho_{2}(C)$ for these generalizations.

The conditional, average conditional and partial multivariate Kendall's tau are then respectively given by

$$
\begin{aligned}
& \tau_{\boldsymbol{Y}}(\boldsymbol{x})=\frac{1}{2^{p-1}-1}\left\{2^{d} \int_{[0,1]^{p}} C_{\boldsymbol{X}}(\boldsymbol{u}) d C_{\boldsymbol{X}}(\boldsymbol{u})-1\right\}, \quad \tau_{\boldsymbol{Y}}^{\mathrm{A}}=\mathrm{E}_{\boldsymbol{X}}\left[\tau_{\boldsymbol{Y}}(\boldsymbol{X})\right], \\
& \bar{\tau}_{\boldsymbol{Y}}=\frac{1}{2^{p-1}-1}\left\{2^{p} \int_{[0,1]^{p}} \bar{C}(\boldsymbol{u}) d \bar{C}(\boldsymbol{u})-1\right\} .
\end{aligned}
$$

\subsection{Multivariate extension of results in Section 3}

Expressions for the average conditional Kendalls'tau and the partial Kendall's tau, similar to these established in Theorem 3.1 can be derived in a similar fashion. However, in the two-dimensional case we used that $\iint_{[0,1]^{2}} C_{1} d C_{2}=\iint_{[0,1]^{2}} C_{2} d C_{1}$. Such a result however only holds in case $p=2$, as was shown in [15, Theorem 3.3, page 67]. Consequently, in the case of general $p$ we cannot simplify $\int_{[0,1]^{p}} C_{1} d C_{2}$ and $\int_{[0,1]^{p}} C_{2} d C_{1}$. In the multivariate case, the expressions analogous to these in Theorem 3.1 are:

$$
\begin{aligned}
& \tau_{\boldsymbol{Y}}^{\mathrm{A}}=\frac{1}{2^{p-1}-1}\left\{2 ^ { p } \left\{\mu_{2} \int_{[0,1]^{p}} C_{1} d C_{1}+\left(\mu_{1}-\mu_{2}\right)\left(\int_{[0,1]^{p}} C_{1} d C_{2}+\int_{[0,1]^{p}} C_{2} d C_{1}\right)\right.\right. \\
& \left.\left.+\left(1-2 \mu_{1}+\mu_{2}\right) \int_{[0,1]^{d}} C_{2} d C_{2}\right\}-1\right\} \\
& \bar{\tau}_{\boldsymbol{Y}}=\frac{1}{2^{p-1}-1}\left\{2 ^ { p } \left\{\mu_{1}^{2} \int_{[0,1]^{p}} C_{1} d C_{1}+\mu_{1}\left(1-\mu_{1}\right)\left(\int_{[0,1]^{p}} C_{1} d C_{2}+\int_{[0,1]^{p}} C_{2} d C_{1}\right)\right.\right. \\
& \left.\left.+\left(1-\mu_{1}\right)^{2} \int_{[0,1]^{p}} C_{2} d C_{2}\right\}-1\right\} \\
& \bar{\tau}_{\boldsymbol{Y}}-\tau_{\boldsymbol{Y}}^{\mathrm{A}}=\frac{2^{p}}{2^{p-1}-1} \operatorname{Var}[w(\boldsymbol{X})] \\
& \times\left\{-\int_{[0,1]^{p}} C_{1} d C_{1}+\int_{[0,1]^{p}} C_{1} d C_{2}+\int_{[0,1]^{p}} C_{2} d C_{1}-\int_{[0,1]^{p}} C_{2} d C_{2}\right\} .
\end{aligned}
$$


Also the result in Corollary 3.1 continuous to hold in the multivariate case, with

$$
T\left(C_{1}, C_{2}\right)=-\int_{[0,1]^{p}} C_{1} d C_{1}+\int_{[0,1]^{p}} C_{1} d C_{2}+\int_{[0,1]^{p}} C_{2} d C_{1}-\int_{[0,1]^{p}} C_{2} d C_{2} .
$$

Using definition (37) for Kendall's tau associated to a $p$-variate copula, it is further seen that $T\left(C_{1}, C_{2}\right)=0$ is equivalent to

$$
\tau\left(\gamma C_{1}+(1-\gamma) C_{2}\right)-\gamma \tau\left(C_{1}\right)-(1-\gamma) \tau\left(C_{2}\right)=0,
$$

for any $\gamma \in[0,1]$. This then leads to an analogous result as in Theorem 3.2.

Not all results in Corollary 3.2 can be generalized to the multivariate setting. Since the lower FréchetHoeffding bound in the $p$-variate case equals $W\left(u_{1}, \ldots, u_{p}\right)=\max \left(\sum_{j=1}^{p} u_{j}-p+1,0\right)$, which is no longer a copula when $p>2$, the third statement in Corollary 3.2 does not have a multivariate analogue. The other two statements of Corollary 3.2 can be extended to the multivariate case. Regarding the first statement, it can been shown, using the generalizations of Spearman's rho in (38) and (39) that

$$
T(C, \Pi)=0 \Longleftrightarrow \tau(C)=\frac{2^{p}-(p+1)}{\left(2^{p-1}-1\right)(p+1)}\left(\rho_{1}(C)+\rho_{2}(C)\right) .
$$

The third statement in Corollary 3.2 involves the upper Fréchet-Hoeffding bound in the $p$-variate case, i.e. $M\left(u_{1}, \ldots, u_{p}\right)=\min \left(u_{1}, \ldots, u_{p}\right)$ which is a copula. When studying $T(C, M)$ one needs to evaluate the integrals $\int_{[0,1]^{p}} M d C$ and $\int_{[0,1]^{p}} C d M$. The latter $p$-dimensional integral reduces to a one-dimensional integral involving the diagonal section of the copula $C$, as pointed out in [15, Example 2.5, page 66]. Furthermore $\int_{[0,1]^{p}} M d M=1 / 2$. All these considerations together lead to

$$
\begin{aligned}
T(C, M) & =-\int_{[0,1]^{p}} C d C+\int_{[0,1]^{p}} C d M+\int_{[0,1]^{p}} M d C-\int_{[0,1]^{p}} M d M \\
& =-\frac{\left(2^{p-1}-1\right) \tau(C)+1}{2^{p}}+\int_{[0,1]^{p}} C(\boldsymbol{u}) d M(\boldsymbol{u})+\int_{[0,1]^{p}} M(\boldsymbol{u}) d C(\boldsymbol{u})-\frac{1}{2} \\
& =-\frac{\left(2^{p-1}-1\right) \tau(C)+1}{2^{p}}+\int_{[0,1]} \delta(u) d u+\int_{[0,1]^{p}} M(\boldsymbol{u}) d C(\boldsymbol{u})-\frac{1}{2} .
\end{aligned}
$$

\subsection{Extension of the results in Section 4}

The proof of Theorem 4.1 relies on Lemma 5.1, which is only valid when $p=2$. However, we can provide an upper bound for the difference in absolute value between the average conditional Kendall's tau and the partial Kendall's tau in case of general $p$. Indeed, [25] also provided an upper bound for the conditional copula as in Lemma 4.1, for general $p$. This upperbound is a $p$-dimensional Clayton copula

$$
M_{1}\left(u_{1}, \ldots, u_{p}\right)=\left(\sum_{j=1}^{p} u_{j}^{-1}-p+1\right)^{-1} .
$$

Kendall's tau for this copula equals

$$
\tau\left(M_{1}\right)=\frac{1}{2^{p-1}-1}\left\{\frac{2^{p}}{(p-1) !}\left[\prod_{k=1}^{p-1}(1+k)\right] \cdot \sum_{k=0}^{p-1}\left(\begin{array}{c}
p-1 \\
k
\end{array}\right) \frac{(-1)^{k}}{k+2}-1\right\},
$$

as was given by [37]. When $p=2$ this reduces to $1 / 3$.

A lower bound for Kendall's tau in dimension $p$ is given by $-1 /\left(2^{p-1}-1\right)$ (see [28]). Applying this bound one then can show that, under condition (17),

$$
\left|\tau^{\mathrm{A}}-\bar{\tau}\right| \leq \frac{1}{2^{p-1}-1}\left\{\frac{2^{p}}{(p-1) !}\left[\prod_{k=1}^{p-1}(1+k)\right] \cdot \sum_{k=0}^{p-1}\left(\begin{array}{c}
p-1 \\
k
\end{array}\right) \frac{(-1)^{k}}{k+2}\right\} .
$$




\subsection{Extension of the results in Section 5.1}

The results of Section 5.1 can be extended to the multivariate case (general $p$ ). For brevity we state the generalizations of the results in Propositions 5.1 and 5.2:

- For two ordered copulas $C_{1}$ and $C_{2}$, it holds that

$$
\left|T\left(C_{1}, C_{2}\right)\right| \leq \frac{2^{p-1}-1}{2^{p}}\left|\tau\left(C_{2}\right)-\tau\left(C_{1}\right)\right| \leq \frac{1}{2} .
$$

* If both copulas describe negative dependence, i.e. $C_{1}, C_{2} \prec \Pi$, then

$$
\left|T\left(C_{1}, C_{2}\right)\right| \leq \frac{2^{p-1}-1}{2^{p}} \frac{1}{2^{p-1}-1}=\frac{1}{2^{p}} .
$$

* If both copulas describe positive dependence, i.e. $C_{1}, C_{2} \succ \Pi$, then

$$
\left|T\left(C_{1}, C_{2}\right)\right| \leq \frac{2^{p-1}-1}{2^{p}} .
$$

- In case of general dimension $p$ one can show that

$$
T(\Pi, C)=\frac{2^{p}-(p+1)}{2^{p}(p+1)}\left(\rho_{1}(C)+\rho_{2}(C)\right)-\frac{\left(2^{p-1}-1\right) \tau(C)}{2^{p}} .
$$

and moreover, relying on upper and lower bounds for the multivariate generalizations of Kendall's tau and Spearman's rho one can show that

$$
\frac{2^{p}-(p+1) !}{2^{p-1}(p+1) p !}-\frac{2^{p-1}-1}{2^{p}} \leq T(\Pi, C) \leq \frac{2^{p}-(p+1)}{2^{p-1}(p+1)}+\frac{1}{2^{p}} .
$$

Further, the results of Proposition 5.3 continue to hold.

\section{Concluding remarks and further discussion}

When studying the dependencies between the components of $\boldsymbol{Y}=\left(Y_{1}, \ldots, Y_{p}\right)^{\mathrm{T}}$ conditionally upon $\boldsymbol{X}=$ $\left(X_{1}, \ldots, X_{d}\right)^{\mathrm{T}}$, one is interested in quantifying the strength of the global dependence between the components, across all realizations of $\boldsymbol{X}$. Examples of such measures of global strength of dependence are the average conditional Kendall's tau and the partial Kendall's tau. Such measures allow, for example, to compare the global strengths of dependencies between the components of $\boldsymbol{Y}=\left(Y_{1}, \ldots, Y_{p}\right)^{\mathrm{T}}$ and components of $\boldsymbol{Z}=\left(Z_{1}, \ldots, Z_{p}\right)^{\mathrm{T}}$, when both dependencies are influenced by the vector of covariates $\boldsymbol{X}$. This paper contributes to this area with a study of the average conditional Kendall's tau and the partial Kendall's tau. Although we mainly focus on the bivariate case (when $p=2$ ), we also discuss extensions to the established results for general $p$ in Section 7.

Kendall's tau is just one example of an association measure. For each given association measure (call it a reference association measure) one can look into the corresponding average conditional association measure and partial association measure. A first interesting question is which properties of the reference association measure imply that both concepts (average conditional association measure and partial association measure) coincide or not. In the bivariate case $(p=2)$ it seems that the answer to this question is determined by the property whether the reference association measure is a linear functional of the copula or not. Among papers that look into such characteristics of reference association measures are [10], [11] and [16]. In the general case $(p \geq 3)$, one should first of all be aware that there are for (some) bivariate association measures several generalizations to the multivariate case. See [17] for a recent discussion of these multivariate association measures, and their specific advantages and disadvantages. Characteristic properties about the type of functionals (of copulas) behind some reference measures of concordance, and consequences of this, are in the general case $(p \geq 3)$ discussed in, for example, [14]. 
The average conditional Kendall's tau and partial Kendall's tau, and other conditional and partial association measures can be looked upon as examples of global measures of strengths of dependencies taking into account influences of covariates. This then also raises questions about orderings between such global measures. A first small study to investigate this for the average conditional Kendall's tau and partial Kendall's tau was provided in Section 6.

\section{Acknowledgement:}

The authors are grateful to the reviewers for their very valuable comments, which led to a considerable improvement of the original manuscript. The authors gratefully acknowledge support of the projects GOA/12/014 and C16/20/002 from the Research Fund KU Leuven. The authors are also very thankful to Professor Fabrizio Durante for helpful discussions.

\section{A Appendix}

\section{A.1 Proofs of theoretical results in Section 3}

\section{A.1.1 Proof of Theorem 3.1}

Note first of all that $\mathrm{E}_{\boldsymbol{X}}\left[w^{\ell}(\boldsymbol{X})\right]<\infty$, with $\ell=1,2$, since $w(\cdot)$ attains values in $[0,1]$.

(i). Starting from the definition of $\tau^{\mathrm{A}}=\mathrm{E}_{\boldsymbol{X}}[\tau(\boldsymbol{X})]$, we obtain

$$
\begin{aligned}
\tau^{\mathrm{A}}=\mathrm{E}_{\boldsymbol{X}}[\tau(\boldsymbol{X})] & =\mathrm{E}_{\boldsymbol{X}}\left[4 \iint_{[0,1]^{2}} C_{\boldsymbol{X}}\left(u_{1}, u_{2}\right) d C_{\boldsymbol{X}}\left(u_{1}, u_{2}\right)-1\right] \\
& =4 \iint_{\mathcal{R}_{\boldsymbol{X}}}\left\{\iint_{[0,1]^{2}} C_{\boldsymbol{X}}\left(u_{1}, u_{2}\right) d C_{\boldsymbol{X}}\left(u_{1}, u_{2}\right)\right\} d F_{\boldsymbol{X}}(\boldsymbol{x})-1 .
\end{aligned}
$$

For the inner integral we get, dropping the writing of the arguments for presentational simplicity

$$
\begin{aligned}
& \iint_{[0,1]^{2}} C_{\boldsymbol{x}}\left(u_{1}, u_{2}\right) d C_{\boldsymbol{x}}\left(u_{1}, u_{2}\right) \\
& =w^{2}(\boldsymbol{x}) \iint_{[0,1]^{2}} C_{1} d C_{1}+2 w(\boldsymbol{x})(1-w(\boldsymbol{x})) \iint_{[0,1]^{2}} C_{1} d C_{2}+(1-w(\boldsymbol{x}))^{2} \iint_{[0,1]^{2}} C_{2} d C_{2},
\end{aligned}
$$

where we used that for any two copulas $C_{1}$ and $C_{2}$, it holds that $\iint C_{1} d C_{2}=\iint C_{2} d C_{1}$ (see e.g. 28, Corollary 5.1.2.). Substituting the above expression for the inner integral into (A.1) leads to

$$
\begin{aligned}
\tau^{\mathrm{A}}= & 4\left\{\iint_{[0,1]^{2}} C_{1} d C_{1} \int_{\mathcal{R}_{\boldsymbol{X}}} w^{2}(\boldsymbol{x}) d F_{\boldsymbol{X}}(\boldsymbol{x})+2 \iint_{[0,1]^{2}} C_{1} d C_{2} \int_{\mathcal{R}_{\boldsymbol{X}}} w(\boldsymbol{x})(1-w(\boldsymbol{x})) d F_{\boldsymbol{X}}(\boldsymbol{x})\right. \\
& \left.+\iint_{[0,1]^{2}} C_{2} d C_{2} \int_{\mathcal{R}_{\boldsymbol{X}}}(1-w(\boldsymbol{x}))^{2} d F_{\boldsymbol{X}}(\boldsymbol{x})\right\}-1 \\
= & 4\left\{\mathrm{E}_{\boldsymbol{X}}\left[w^{2}(\boldsymbol{X})\right] \iint_{[0,1]^{2}} C_{1} d C_{1}+2 \mathrm{E}_{\boldsymbol{X}}[w(\boldsymbol{X})(1-w(\boldsymbol{X}))] \iint_{[0,1]^{2}} C_{1} d C_{2}\right. \\
& \left.+\mathrm{E}_{\boldsymbol{X}}\left[(1-w(\boldsymbol{X}))^{2}\right] \iint_{[0,1]^{2}} C_{2} d C_{2}\right\}-1 .
\end{aligned}
$$


Since $\mathrm{E}_{\boldsymbol{X}}\left[w^{2}(\boldsymbol{X})\right]=\mu_{2}, \mathrm{E}_{\boldsymbol{X}}[w(\boldsymbol{X})(1-w(\boldsymbol{X}))]=\mu_{1}-\mu_{2}$ and $\mathrm{E}_{\boldsymbol{X}}\left[(1-w(\boldsymbol{X}))^{2}\right]=1-2 \mu_{1}+\mu_{2}$, the statement follows.

(ii). Starting from the definition of $\bar{\tau}$ we obtain

$$
\begin{aligned}
\bar{\tau} & =4 \iint_{[0,1]^{2}} \mathrm{E}_{\boldsymbol{X}}\left[C_{\boldsymbol{X}}\left(u_{1}, u_{2}\right)\right] d \mathrm{E}_{\boldsymbol{X}}\left[C_{\boldsymbol{X}}\left(u_{1}, u_{2}\right)\right]-1 \\
& =4 \iiint_{[0,1]^{2}} \int_{\mathcal{R}_{\boldsymbol{X}}} C_{\boldsymbol{X}}\left(u_{1}, u_{2}\right) d F_{\boldsymbol{X}}(\boldsymbol{x}) d \boldsymbol{u}\left[\int_{\mathcal{R}_{\boldsymbol{X}}} C_{\boldsymbol{X}^{\prime}}\left(u_{1}, u_{2}\right) d F_{\boldsymbol{X}}\left(\boldsymbol{x}^{\prime}\right)\right]-1 \\
& =4 \iint_{\mathcal{R}_{\boldsymbol{X}}} \int_{\mathcal{R}_{\boldsymbol{X}}}\left\{\iint_{[0,1]^{2}} C_{\boldsymbol{X}}\left(u_{1}, u_{2}\right) d C_{\boldsymbol{X}^{\prime}}\left(u_{1}, u_{2}\right)\right\} d F_{\boldsymbol{X}}(\boldsymbol{x}) d F_{\boldsymbol{X}}\left(\boldsymbol{x}^{\prime}\right)-1,
\end{aligned}
$$

where we denoted $d_{\boldsymbol{u}}$ the differential with respect to $\boldsymbol{u}$. Similarly as to (A.2) the inner integral is

$$
\begin{aligned}
\iint_{[0,1]^{2}} C_{\boldsymbol{x}}\left(u_{1}, u_{2}\right) d C_{\boldsymbol{x}^{\prime}}\left(u_{1}, u_{2}\right)= & w(\boldsymbol{x}) w\left(\boldsymbol{x}^{\prime}\right) \iint_{[0,1]^{2}} C_{1} d C_{1} \\
& +\left[w\left(\boldsymbol{x}^{\prime}\right)(1-w(\boldsymbol{x}))+w(\boldsymbol{x})\left(1-w\left(\boldsymbol{x}^{\prime}\right)\right)\right] \iint_{[0,1]^{2}} C_{1} d C_{2} \\
& +(1-w(\boldsymbol{x}))\left(1-w\left(\boldsymbol{x}^{\prime}\right)\right) \iint_{[0,1]^{2}} C_{2} d C_{2},
\end{aligned}
$$

which after substitution into (A.3) gives

$$
\begin{gathered}
\bar{\tau}=4\left\{\left(\mathrm{E}_{\boldsymbol{X}}[w(\boldsymbol{X})]\right)^{2} \iint_{[0,1]^{2}} C_{1} d C_{1}+2 \mathrm{E}_{\boldsymbol{X}}[w(\boldsymbol{X})]\left(1-\mathrm{E}_{\boldsymbol{X}}[w(\boldsymbol{X})]\right) \iint_{[0,1]^{2}} C_{1} d C_{2}\right. \\
\left.+\left(1-\mathrm{E}_{\boldsymbol{X}}[w(\boldsymbol{X})]\right)^{2} \iint_{[0,1]^{2}} C_{2} d C_{2}\right\}-1,
\end{gathered}
$$

which leads to the statement in (ii).

(iii). This immediately follows from the expressions in (i) and (ii).

\section{A.1.2 Proof of Theorem 3.2}

We start by looking at an arbitrary linear combination of two arbitrary copulas $C_{1}$ and $C_{2}$. More precisely given two copulas $C_{1}$ and $C_{2}$ consider

$$
C\left(u_{1}, u_{2}\right)=\gamma C_{1}\left(u_{1}, u_{2}\right)+(1-\gamma) C_{2}\left(u_{1}, u_{2}\right) \quad \text { with } \gamma \in[0,1]
$$

First of all note that this is a copula for all $\gamma \in[0,1]$. In the special cases that $\gamma=0$ or $\gamma=1$, the copula $C=C_{2}$ or $C=C_{1}$. 
We next calculate Kendall's tau for such an arbitrary linear combination. Denote by $\tau(C), \tau\left(C_{1}\right), \tau\left(C_{2}\right)$, Kendall's tau associated to respectively the copulas $C, C_{1}$ and $C_{2}$. We find

$$
\begin{aligned}
\tau(C)= & 4 \iint C d C-1 \\
= & 4 \iint\left(\gamma C_{1}+(1-\gamma) C_{2}\right) d\left(\gamma C_{1}+(1-\gamma) C_{2}\right)-1 \\
= & \left.\gamma^{2}\left\{4 \iint C_{1} d C_{1}-1\right\}+(1-\gamma)^{2}\left\{4 \iint C_{2} d C_{2}-1\right\}+2 \gamma(1-\gamma)\left\{4 \iint C_{1} d C_{2}-1\right)\right\} \\
& \quad+\left(-1+\gamma^{2}+(1-\gamma)^{2}+2 \gamma(1-\gamma)\right) \\
= & \gamma^{2} \tau\left(C_{1}\right)+(1-\gamma)^{2} \tau\left(C_{2}\right)+2 \gamma(1-\gamma)\left\{4 \iint C_{1} d C_{2}-1\right\} .
\end{aligned}
$$

Expression (A.5) in fact shows that $\tau(C)$, Kendall's tau of the linear combination of $C_{1}$ and $C_{2}$ is a quadratic function in $\gamma$. See [11, Definition 2] for the concept of the degree of an association measure.

For the special case that $C_{1}$ and $C_{2}$ are both Archimedean copulas, a proof of the above statement can be found in [4].

From (A.5) and (6) we then obtain that, for all $\gamma \in(0,1)$,

$$
\begin{aligned}
T\left(C_{1}, C_{2}\right) & =-\iint_{[0,1]^{2}} C_{1} d C_{1}+2 \iint_{[0,1]^{2}} C_{1} d C_{2}-\iint_{[0,1]^{2}} C_{2} d C_{2} \\
& =-\frac{\tau\left(C_{1}\right)+1}{4}-\frac{\tau\left(C_{2}\right)+1}{4}+\frac{\tau(C)-\gamma^{2} \tau\left(C_{1}\right)-(1-\gamma)^{2} \tau\left(C_{2}\right)+2 \gamma(1-\gamma)}{4 \gamma(1-\gamma)} \\
& =\frac{\tau(C)-\gamma \tau\left(C_{1}\right)-(1-\gamma) \tau\left(C_{2}\right)}{4 \gamma(1-\gamma)} .
\end{aligned}
$$

Hence $T\left(C_{1}, C_{2}\right)=0$ is equivalent to

$$
\tau(C)-\gamma \tau\left(C_{1}\right)-(1-\gamma) \tau\left(C_{2}\right)=0 \quad \forall \gamma \in[0,1],
$$

since also for $\gamma=0$ (i.e. $C=C_{2}$ ) and $\gamma=1$ (i.e. $C=C_{1}$ ) the equality holds. This proves the statement.

\section{A.1.3 Proof of Corollary 3.2}

Although all statements in Corollary 3.2 can be proven starting from the equivalence (ii) in Theorem 3.2, it is simpler to directly start from the definition of the quantity $T\left(C_{1}, C_{2}\right)$.

1). Consider $C_{2}=\Pi$, and denote $C_{1}=C$. We are then curious to know which are the conditions that $C$ needs to satisfy for having that $T\left(C_{1}, C_{2}\right)=0$. Recalling (6), we obtain that

$$
\begin{aligned}
T(C, \Pi)=0 & \Longleftrightarrow-\iint C d C+2 \iint C d \Pi-\iint \Pi d \Pi=0 \\
& \Longleftrightarrow-\iint C d C+2 \iint C d \Pi-\frac{1}{4}=0 \\
& \Longleftrightarrow 4 \iint C d C=8 \iint C d \Pi-1 \\
& \Longleftrightarrow \tau(C)+1=2\left(\frac{1}{3} \rho(C)-1\right)-1 \\
& \Longleftrightarrow \tau(C)=\frac{2}{3} \rho(C) .
\end{aligned}
$$

2). Consider $C_{2}=M$, and denote $C_{1}=C$. For this case we get

$$
T(C, M)=-\iint C d C+2 \iint C d M-\iint M d M=-\iint C d C+2 \iint C d M-\frac{1}{2},
$$


since $\iint M d M=1 / 2$. See, for example, Section 5.1 in [28].

Now, for any copula $C$ that is integrable we have that

$$
\iint C d M=\int_{0}^{1} C(u, u) d u=\int_{0}^{1} \delta(u) d u,
$$

with $\delta(u)=C(u, u)$ the diagonal section of the copula $C$. See [28, Example 5.1, page 160]. Using this fact, we can write

$$
\begin{aligned}
T(C, M)=0 \Longleftrightarrow 2 \int_{0}^{1} \delta(u) d u=\iint C d C+\frac{1}{2} & \Longleftrightarrow 2 \int_{0}^{1} \delta(u) d u=\frac{\tau(C)+1}{4}+\frac{1}{2} \\
& \Longleftrightarrow \int_{0}^{1} \delta(u) d u=\frac{\tau(C)+3}{8} .
\end{aligned}
$$

This finished the proof of 2).

3). In case $C_{2}=W$, and $C_{1}=C$ we find

$$
\begin{aligned}
T(C, W) & =-\iint C d C+2 \iint C d W-\iint W d W \\
& =-\iint C d C+2 \int C(u, 1-u) d u-0 \\
& =-\frac{\tau(C)+1}{4}+2 \int C(u, 1-u) d u
\end{aligned}
$$

and hence $T(C, W)=0$ leads to

$$
\frac{\tau(C)+1}{4}=2 \int C(u, 1-u) d u
$$

\section{A.2 Proof of Theorem 4.1 in Section 4}

Using Lemma 5.1 we get that

$$
\begin{aligned}
\bar{\tau} & =4 \iint_{\mathcal{R}_{X}} \int_{\mathcal{R}_{X}} \iint_{[0,1]^{2}} C_{X}\left(u_{1}, u_{2}\right) d C_{x^{\prime}}\left(u_{1}, u_{2}\right) d F_{X}(x) d F_{X}\left(x^{\prime}\right)-1 \\
& =1-4 \iint_{\mathcal{R}_{X}} \iint_{\mathcal{R}_{X}} \frac{\partial C_{X}\left(u_{1}, u_{2}\right)}{\partial u_{1}} \frac{\partial C_{X^{\prime}}\left(u_{1}, u_{2}\right)}{\partial u_{2}} d u_{1} d u_{2} d F_{X}(x) d F_{X}\left(x^{\prime}\right) \\
& =1-4 \iiint \int_{[0,1]^{4}} g\left(w, u_{1}, u_{2}\right) \cdot g\left(w^{\prime}, u_{2}, u_{1}\right) d u_{1} d u_{2} d w d w^{\prime} \\
& =1-4 \iint_{[0,1]^{2}}\left\{\int_{[0,1]} g\left(w, u_{1}, u_{2}\right) d w\right\}\left\{\int_{[0,1]} g\left(w, u_{2}, u_{1}\right) d w\right\} d u_{1} d u_{2} .
\end{aligned}
$$

The average conditional Kendall's tau equals

$$
\begin{aligned}
\tau^{\mathrm{A}} & =4 \iint_{\mathcal{R}_{X}} \iint_{[0,1]^{2}} C_{X}\left(u_{1}, u_{2}\right) d C_{\chi}\left(u_{1}, u_{2}\right) d F_{X}(x)-1 \\
& =1-4 \iint_{\mathcal{R}_{X}} \iint_{[0,1]^{2}} \frac{\partial C_{x}\left(u_{1}, u_{2}\right)}{\partial u_{1}} \frac{\partial C_{X}\left(u_{1}, u_{2}\right)}{\partial u_{2}} d u_{1} d u_{2} d F_{X}(x) \\
& =1-4 \iint_{[0,1]^{2}}\left\{\int_{[0,1]} g\left(w, u_{1}, u_{2}\right) g\left(w, u_{2}, u_{1}\right) d w\right\} d u_{1} d u_{2} .
\end{aligned}
$$


So we can see that when

$$
\left\{\int_{[0,1]} g\left(w, u_{1}, u_{2}\right) d w\right\}\left\{\int_{[0,1]} g\left(w, u_{2}, u_{1}\right) d w\right\}=\int_{[0,1]} g\left(w, u_{1}, u_{2}\right) g\left(w, u_{2}, u_{1}\right) d w
$$

this implies that $\bar{\tau}=\tau^{\mathrm{A}}$.

From Lemma 4.1 we know that under Condition (17), the conditional Archimedean copula $C_{\boldsymbol{x}}\left(u_{1}, u_{2}\right)$ is bounded above by the copula $M_{1}\left(u_{1}, u_{2}\right)=\frac{u_{1} u_{2}}{u_{1}+u_{2}-u_{1} u_{2}}$. Kendall's tau of the latter equals $1 / 3$ (see [25]). This means that

$$
\tau^{\mathrm{A}}=\mathrm{E}_{\boldsymbol{X}}[\tau(\boldsymbol{X})] \leq \mathrm{E}_{\boldsymbol{X}}\left[\tau_{M_{1}}\right]=\tau_{M_{1}}=\frac{1}{3} .
$$

For the partial Kendall's tau we find the same bound:

$$
\bar{\tau}=4 \iint \bar{C}\left(u_{1}, u_{2}\right) d \bar{C}\left(u_{1}, u_{2}\right)-1 \leq 4 \iint M_{1}\left(u_{1}, u_{2}\right) d M_{1}\left(u_{1}, u_{2}\right)-1=\tau_{M_{1}}=\frac{1}{3},
$$

where we used that $\bar{C}=\mathrm{E}_{\boldsymbol{X}}\left[C_{\boldsymbol{X}}\right] \leq \mathrm{E}_{\boldsymbol{X}}\left[M_{1}\right]=M_{1}$.

This results into an upperbound for $\left|\tau^{\mathrm{A}}-\bar{\tau}\right|$ of $4 / 3$.

\section{A.3 Proofs of theoretical results in Section 5}

\section{A.3.1 Proof of Proposition 5.1}

Under the assumption of positively ordered copulas, i.e. $C_{1} \prec C_{2}$, we can establish the following upper bound for $\left|T\left(C_{1}, C_{2}\right)\right|$. On the one hand we have that

$$
\begin{aligned}
T\left(C_{1}, C_{2}\right) & =-\iint_{[0,1]^{2}} C_{1} d C_{1}+2 \iint_{[0,1]^{2}} C_{1} d C_{2}-\iint_{[0,1]^{2}} C_{2} d C_{2} \\
& =-\frac{\tau\left(C_{1}\right)+1}{4}+2 \iint_{[0,1]^{2}} C_{1} d C_{2}-\frac{\tau\left(C_{2}\right)+1}{4} \\
& \leq-\frac{\tau\left(C_{1}\right)+\tau\left(C_{2}\right)}{4}+2 \iint_{[0,1]^{2}} C_{2} d C_{2}-\frac{1}{2} \\
& =-\frac{\tau\left(C_{1}\right)+\tau\left(C_{2}\right)}{4}+2 \cdot \frac{\tau\left(C_{2}\right)+1}{4}-\frac{1}{2} \\
& =\frac{\tau\left(C_{2}\right)-\tau\left(C_{1}\right)}{4} .
\end{aligned}
$$

On the other hand we get

$$
\begin{aligned}
T\left(C_{1}, C_{2}\right) & =-\frac{\tau\left(C_{1}\right)+1}{4}+2 \iint_{[0,1]^{2}} C_{1} d C_{2}-\frac{\tau\left(C_{2}\right)+1}{4} \\
& \geq-\frac{\tau\left(C_{1}\right)+\tau\left(C_{2}\right)}{4}+2 \iint_{[0,1]^{2}} C_{1} d C_{1}-\frac{1}{2} \\
& =-\frac{\tau\left(C_{1}\right)+\tau\left(C_{2}\right)}{4}+2 \cdot \frac{\tau\left(C_{1}\right)+1}{4}-\frac{1}{2} \\
& =\frac{\tau\left(C_{1}\right)-\tau\left(C_{2}\right)}{4}
\end{aligned}
$$


Consequently,

$$
\left|T\left(C_{1}, C_{2}\right)\right| \leq \frac{\tau\left(C_{2}\right)-\tau\left(C_{1}\right)}{4} \leq \frac{1}{2}, \quad \text { for } C_{1} \prec C_{2} \text {. }
$$

Similarly, if $C_{1} \succ C_{2}$, then

$$
\left|T\left(C_{1}, C_{2}\right)\right| \leq \frac{\tau\left(C_{1}\right)-\tau\left(C_{2}\right)}{4} \leq \frac{1}{2} .
$$

In summary, for two ordered copulas it holds that

$$
\left|T\left(C_{1}, C_{2}\right)\right| \leq \frac{\left|\tau\left(C_{2}\right)-\tau\left(C_{1}\right)\right|}{4} \leq \frac{1}{2} .
$$

If we additionally assume that both copulas describe negative dependence, i.e.

$$
C_{1}, C_{2} \prec \Pi \text {, }
$$

then we have that $\max \left(\tau\left(C_{1}\right), \tau\left(C_{2}\right)\right) \leq 0$, and consequently

$$
\left|T\left(C_{1}, C_{2}\right)\right| \leq \frac{\left|\tau\left(C_{2}\right)-\tau\left(C_{1}\right)\right|}{4} \leq \frac{1}{4} .
$$

If both copulas describe positive dependence,

$$
C_{1}, C_{2} \succ \Pi
$$

then $\min \left(\tau\left(C_{1}\right), \tau\left(C_{2}\right)\right) \geq 0$, and the same holds.

\section{A.3.2 Proof of Proposition 5.2}

We need to evaluate $|T(\Pi, C)|$, where $C$ is any arbitrary copula. Note that

$$
\begin{aligned}
|T(\Pi, C)|=\left|-\iint_{[0,1]^{2}} \Pi d \Pi+2 \iint_{[0,1]^{2}} C d \Pi-\iint_{[0,1]^{2}} C d C\right| & =\left|-\frac{1}{4}+2 \cdot \frac{\rho(C)+3}{12}-\frac{\tau(C)+1}{4}\right| \\
& =\frac{|2 \rho(C)-3 \tau(C)|}{12} .
\end{aligned}
$$

Theorem 5.1.10 on page 175 in [28] gives that

$$
-1 \leq 2 \rho(C)-3 \tau(C) \leq 1,
$$

which finishes the proof.

\section{A.3.3 Proof of Proposition 5.3}

(1) Consider a positively ordered family of copulas. Since $B=\left[\theta_{1}^{\prime}, \theta_{2}^{\prime}\right]$ we then know that for all $\theta_{1}^{\prime}, \theta_{1}, \theta_{2}, \theta_{2}^{\prime} \in \Theta$ for which $\theta_{1}^{\prime} \leq \theta_{1} \leq \theta_{2} \leq \theta_{2}^{\prime}$, it holds that $C_{1}^{\prime} \prec C_{1} \prec C_{2} \prec C_{2}^{\prime}$.

(a). If $k$ is supermodular, then for $\theta_{1} \leq \theta_{2}$, taking $x_{1}=\theta_{2}=y_{2}, x_{2}=\theta_{1}=y_{1}$ in (23), we find that $k\left(\theta_{1}, \theta_{2}\right)-k\left(\theta_{1}, \theta_{1}\right) \leq k\left(\theta_{2}, \theta_{2}\right)-k\left(\theta_{2}, \theta_{1}\right)$. This is equivalent with $T\left(\theta_{1}, \theta_{2}\right) \leq 0$.

The upperbound for $\left|T\left(C_{1}, C_{2}\right)\right|$ can be obtained as follows

$$
\begin{aligned}
\left|T\left(C_{1}, C_{2}\right)\right| & =\left(k\left(\theta_{2}, \theta_{2}\right)-k\left(\theta_{2}, \theta_{1}\right)\right)-\left(k\left(\theta_{1}, \theta_{2}\right)-k\left(\theta_{1}, \theta_{1}\right)\right) \\
& \leq\left(k\left(\theta_{2}^{\prime}, \theta_{2}\right)-k\left(\theta_{2}^{\prime}, \theta_{1}\right)\right)-\left(k\left(\theta_{1}^{\prime}, \theta_{2}\right)-k\left(\theta_{1}^{\prime}, \theta_{1}\right)\right) \\
& =\left(k\left(\theta_{2}^{\prime}, \theta_{2}\right)-k\left(\theta_{1}^{\prime}, \theta_{2}\right)\right)-\left(k\left(\theta_{2}^{\prime}, \theta_{1}\right)-k\left(\theta_{1}^{\prime}, \theta_{1}\right)\right) \\
& \leq\left(k\left(\theta_{2}^{\prime}, \theta_{2}^{\prime}\right)-k\left(\theta_{1}^{\prime}, \theta_{2}^{\prime}\right)\right)-\left(k\left(\theta_{2}^{\prime}, \theta_{1}^{\prime}\right)-k\left(\theta_{1}^{\prime}, \theta_{1}^{\prime}\right)\right) \\
& =\left|T\left(C_{1}^{\prime}, C_{2}^{\prime}\right)\right|,
\end{aligned}
$$


where we used four times the definition of supermodularity by taking respectively $\left(y_{1}=y_{2}=\theta_{2}, x_{1}=\right.$ $\left.\theta_{2}^{\prime}, x_{2}=\theta_{1}\right),\left(y_{1}=\theta_{1}^{\prime}, y_{2}=\theta_{2}, x_{1}=x_{2}=\theta_{1}\right),\left(y_{1}=\theta_{2}^{\prime}=x_{2}, y_{2}=\theta_{2}, x_{1}=\theta_{1}^{\prime}\right)$ and $\left(y_{1}=\theta_{2}^{\prime}, y_{2}=\theta_{1}^{\prime}=\right.$ $\left.x_{1}, x_{2}=\theta_{1}\right)$.

(b). If $k$ is submodular, $-k$ is supermodular, and it follows immediately that $T\left(\theta_{1}, \theta_{2}\right) \geq 0$. And in a similar way as above it can be shown that $\left|T\left(C_{1}, C_{2}\right)\right| \leq\left|T\left(C_{1}^{\prime}, C_{2}^{\prime}\right)\right|$.

(2) Consider now a negatively ordered family of copulas. For all $\theta_{1}^{\prime}, \theta_{1}, \theta_{2}, \theta_{2}^{\prime} \in \Theta$ for which $\theta_{2}^{\prime} \leq \theta_{2} \leq \theta_{1} \leq$ $\theta_{1}^{\prime}$, it holds that $C_{1}^{\prime} \prec C_{1} \prec C_{2} \prec C_{2}^{\prime}$. Similar arguments as in part (1) lead to the results.

\section{A.4 Proofs of theoretical results in Section 6}

\section{A.4.1 Proof Theorem 6.1}

Before proving parts (i) - (iii) of the theorem, we provide some preliminary considerations. From

$$
\begin{aligned}
& \tau_{\boldsymbol{Y}}^{\mathrm{A}}=4\left[\mu_{2} \iint C_{1} d C_{1}+2\left(\mu_{1}-\mu_{2}\right) \iint C_{1} d C_{2}+\left(1-2 \mu_{1}+\mu_{2}\right) \iint C_{2} d C_{2}\right]-1, \\
& \tau_{Z}^{\mathrm{A}}=4\left[\mu_{2} \iint C_{3} d C_{3}+2\left(\mu_{1}-\mu_{2}\right) \iint C_{3} d C_{4}+\left(1-2 \mu_{1}+\mu_{2}\right) \iint C_{4} d C_{4}\right]-1, \\
& \bar{\tau}_{\boldsymbol{Y}}=4\left[\mu_{1}^{2} \iint C_{1} d C_{1}+2\left(\mu_{1}-\mu_{1}^{2}\right) \iint C_{1} d C_{2}+\left(1-2 \mu_{1}+\mu_{1}^{2}\right) \iint C_{2} d C_{2}\right]-1, \\
& \bar{\tau}_{\boldsymbol{Z}}=4\left[\mu_{1}^{2} \iint C_{3} d C_{3}+2\left(\mu_{1}-\mu_{1}^{2}\right) \iint C_{3} d C_{4}+\left(1-2 \mu_{1}+\mu_{1}^{2}\right) \iint C_{4} d C_{4}\right]-1 .
\end{aligned}
$$

it is easy to see that

$$
\begin{aligned}
& \tau_{\boldsymbol{Y}}^{\mathrm{A}} \leq \tau_{\boldsymbol{Z}}^{\mathrm{A}} \\
& \Longleftrightarrow-\mu_{2} T\left(C_{1}, C_{2}\right)+2 \mu_{1}\left(\iint C_{1} d C_{2}-\iint C_{2} d C_{2}\right)+\iint C_{2} d C_{2} \\
& \leq-\mu_{2} T\left(C_{3}, C_{4}\right)+2 \mu_{1}\left(\iint C_{3} d C_{4}-\iint C_{4} d C_{4}\right)+\iint C_{4} d C_{4} \\
& \Longleftrightarrow \begin{cases}\mu_{2} \geq \frac{2 \mu_{1} b-\iint C_{4} d C_{4}+\iint C_{2} d C_{2}}{T\left(C_{1}, C_{2}\right)-T\left(C_{3}, C_{4}\right)}, & \text { if } T\left(C_{1}, C_{2}\right)>T\left(C_{3}, C_{4}\right) \\
\mu_{2} \leq \frac{2 \mu_{1} b-\iint C_{4} d C_{4}+\iint C_{2} d C_{2}}{T\left(C_{1}, C_{2}\right)-T\left(C_{3}, C_{4}\right)}, & \text { if } T\left(C_{1}, C_{2}\right)<T\left(C_{3}, C_{4}\right),\end{cases}
\end{aligned}
$$

and

$$
\begin{aligned}
& \bar{\tau}_{\boldsymbol{Y}}>\bar{\tau}_{\boldsymbol{Z}} \\
& \Longleftrightarrow-\mu_{1}^{2} T\left(C_{1}, C_{2}\right)+2 \mu_{1}\left(\iint C_{1} d C_{2}-\iint C_{2} d C_{2}\right)+\iint C_{2} d C_{2} \\
& \quad>-\mu_{1}^{2} T\left(C_{3}, C_{4}\right)+2 \mu_{1}\left(\iint C_{3} d C_{4}-\iint C_{4} d C_{4}\right)+\iint C_{4} d C_{4} \\
& \Longleftrightarrow\left(T\left(C_{1}, C_{2}\right)-T\left(C_{3}, C_{4}\right)\right) \mu_{1}^{2}+2\left(\iint C_{3} d C_{4}-\iint C_{4} d C_{4}-\iint C_{1} d C_{2}+\iint C_{2} d C_{2}\right) \mu_{1} \\
& \quad+\iint C_{4} d C_{4}-\iint C_{2} d C_{2}<0 .
\end{aligned}
$$


The latter inequality is quadratic in $\mu_{1}$. Given the expressions in (A.6) and (A.7) it is clear that we need to focus on the signs of the quantities $T\left(C_{1}, C_{2}\right)-T\left(C_{3}, C_{4}\right)$ and $D:=\left(\iint C_{3} d C_{4}-\iint C_{1} d C_{2}\right)^{2}-$ $\left(\iint C_{3} d C_{3}-\iint C_{1} d C_{1}\right)\left(\iint C_{4} d C_{4}-\iint C_{2} d C_{2}\right)$.

Proof of part (i).

Note first of all that when $T\left(C_{1}, C_{2}\right)-T\left(C_{3}, C_{4}\right)>0$, the function in (A.7) is convex. It then only remains to discuss the possible roots of the quadratic equation in (A.7). If $D>0$, equation (A.7) has two roots, namely

$$
\mu_{1}=\frac{b \pm \sqrt{D}}{T\left(C_{1}, C_{2}\right)-T\left(C_{3}, C_{4}\right)} .
$$

Since the function in (A.7) is convex, inequality (A.7) holds when

$$
\frac{b-\sqrt{D}}{T\left(C_{1}, C_{2}\right)-T\left(C_{3}, C_{4}\right)}<\mu_{1}<\frac{b+\sqrt{D}}{T\left(C_{1}, C_{2}\right)-T\left(C_{3}, C_{4}\right)} .
$$

If $D \leq 0$ then the function in (A.7) is convex and positive, so inequality (A.7) would never hold.

Proof of part (ii).

Similarly, when $T\left(C_{1}, C_{2}\right)-T\left(C_{3}, C_{4}\right)<0$, the function in (A.7) is concave and so the inequality (A.7) holds when

$$
\mu_{1}<\frac{b+\sqrt{D}}{T\left(C_{1}, C_{2}\right)-T\left(C_{3}, C_{4}\right)} . \quad \text { and } \quad \mu_{1}>\frac{b-\sqrt{D}}{T\left(C_{1}, C_{2}\right)-T\left(C_{3}, C_{4}\right)} .
$$

The various statements of this part then follow immediately.

Proof of part (iii).

If finally $T\left(C_{1}, C_{2}\right)-T\left(C_{3}, C_{4}\right)=0$, then

$$
\tau_{\boldsymbol{Y}}^{\mathrm{A}} \leq \tau_{\boldsymbol{Z}}^{\mathrm{A}} \Longleftrightarrow-2 \mu_{1} b+\iint C_{4} d C_{4}-\iint C_{2} d C_{2} \geq 0
$$

and

$$
\bar{\tau}_{\boldsymbol{Y}}>\bar{\tau}_{b Z} \Longleftrightarrow-2 \mu_{1} b+\iint C_{4} d C_{4}-\iint C_{2} d C_{2}<0,
$$

so it cannot happen that both $\tau_{\boldsymbol{Y}}^{\mathrm{A}} \leq \tau_{\boldsymbol{Z}}^{\mathrm{A}}$ and $\bar{\tau}_{\boldsymbol{Y}}>\bar{\tau}_{\boldsymbol{Z}}$.

A feasible condition for $D \geq 0$ is $C_{3} \prec C_{1}$ and $C_{2} \prec C_{4}$ (or $C_{1} \prec C_{3}$ and $C_{4} \prec$ $C_{2}$ ). Indeed, this condition would imply $\iint C_{3} d C_{3} \leq \iint C_{1} d C_{1}$ and $\iint C_{2} d C_{2} \leq \iint C_{4} d C_{4}$ causing $\left(\iint C_{3} d C_{3}-\iint C_{1} d C_{1}\right)\left(\iint C_{4} d C_{4}-\iint C_{2} d C_{2}\right) \leq 0$, and consequently $D \geq 0$.

\section{A.4.2 Proof of Corollary 6.1}

If $T\left(C_{1}, C_{2}\right)-T\left(C_{3}, C_{4}\right)>0$, we have the following

$$
\begin{aligned}
\bar{\tau}_{\boldsymbol{Y}} \leq \bar{\tau}_{\boldsymbol{Z}} & \Longleftrightarrow\left(T\left(C_{1}, C_{2}\right)-T\left(C_{3}, C_{4}\right)\right) \mu_{1}^{2}-2 \mu_{1} b+\iint C_{4} d C_{4}-\iint C_{2} d C_{2} \geq 0 \\
& \Longleftrightarrow \mu_{1}^{2} \geq \frac{2 \mu_{1} b-\iint C_{4} d C_{4}+\iint C_{2} d C_{2}}{T\left(C_{1}, C_{2}\right)-T\left(C_{3}, C_{4}\right)} .
\end{aligned}
$$

Since $\mu_{1}$ and $\mu_{2}$ are respectively the first and second moment of $w(X)$, it holds that $\mu_{2} \geq \mu_{1}^{2}$. So we also have that

$$
\mu_{2} \geq \frac{2 \mu_{1} b-\iint C_{4} d C_{4}+\iint C_{2} d C_{2}}{T\left(C_{1}, C_{2}\right)-T\left(C_{3}, C_{4}\right)},
$$

which is equivalent to $\tau_{\boldsymbol{Y}}^{\mathrm{A}} \leq \tau_{\boldsymbol{Z}}^{\mathrm{A}}$.

Moreover, if $D \leq 0$, then we know from Theorem 6.1 that $\bar{\tau}_{\mathbf{Y}} \leq \bar{\tau}_{\mathbf{Z}}$ for all $\mu_{1} \in[0,1]$, so we can conclude that also $\tau_{\boldsymbol{Y}}^{\mathrm{A}} \leq \tau_{\boldsymbol{Z}}^{\mathrm{A}}$ is true. 
If on the other hand $T\left(C_{1}, C_{2}\right)-T\left(C_{3}, C_{4}\right)<0$, then

$$
\bar{\tau}_{\boldsymbol{Y}} \geq \bar{\tau}_{\boldsymbol{Z}} \Longleftrightarrow \mu_{1}^{2} \geq \frac{2 \mu_{1} b-\iint C_{4} d C_{4}+\iint C_{2} d C_{2}}{T\left(C_{1}, C_{2}\right)-T\left(C_{3}, C_{4}\right)} .
$$

Similarly we get that

$$
\bar{\tau}_{\boldsymbol{Y}} \geq \bar{\tau}_{\boldsymbol{Z}} \Rightarrow \mu_{2} \geq \frac{2 \mu_{1} b-\iint C_{4} d C_{4}+\iint C_{2} d C_{2}}{T\left(C_{1}, C_{2}\right)-T\left(C_{3}, C_{4}\right)},
$$

which is equivalent to $\tau_{\boldsymbol{Y}}^{\mathrm{A}} \geq \tau_{\boldsymbol{Z}}^{\mathrm{A}}$.

Additionally assuming that $D<0$ means that $\bar{\tau}_{\boldsymbol{Y}}>\bar{\tau}_{\boldsymbol{Z}}$ according to Theorem 6.1, which implies that also $\tau_{\boldsymbol{Y}}^{\mathrm{A}}>\tau_{\boldsymbol{Z}}^{\mathrm{A}}$

Supplementary Material: The online version of this article: (DOI: https://doi.org/10.1515/demo-2021-0104) offers supplementary material.

\section{References}

[1] Acar, E. F., C. Genest, and J. Nešlehová (2012). Beyond simplified pair-copula constructions. J. Multivariate Anal. 110, 74-90.

[2] Bergsma, W. P. (2004). Testing conditional independence for continuous random variables. Available at https://www. eurandom.tue.nl/reports/2004/048-report.pdf.

[3] Bergsma, W. P. (2011). Nonparametric testing of conditional independence by means of the partial copula. Available at https://arxiv.org/abs/1101.4607.

[4] Caillault, C. and D. Guégan (2005). Empirical estimation of tail dependence using copulas: application to Asian markets. Quant. Finance 5(5), 489-501.

[5] Cherubini, U., E. Luciano, and W. Vecchiato (2004). Copula Methods in Finance. John Wiley \& Sons, Chichester.

[6] De Amo, E., H. De Meyer, M. Díaz Carrillo, and J. Fernández-Sánchez (2016). Characterization of copulas with given diagonal and opposite diagonal sections. Fuzzy Set. Syst. 284, 63-77.

[7] De Baets, B., H. De Meyer, and M. Úbeda-Flores (2011). Constructing copulas with given diagonal and opposite diagonal sections. Comm. Statist. Theory Methods 40(5), 828-843.

[8] Derumigny, A. and J.-D. Fermanian (2017). About tests of the "simplifying" assumption for conditional copulas. Depend. Model. 5, 154-197.

[9] Durante, F. and C. Sempi (2016). Principles of Copula Theory. CRC Press, Boca Raton FL.

[10] Edwards, H. H., P. Mikusiński, and M. D. Taylor (2005). Measures of concordance determined by $D_{4}$-invariant measures on $(0,1)^{2}$. Proc. Amer. Math. Soc. 133(5), 1505-1513.

[11] Edwards, H. H. and M. D. Taylor (2009). Characterizations of degree one bivariate measures of concordance. J. Multivariate Anal. 100(8), 1777-1791.

[12] Fernández-Sánchez, J. and M. Úbeda-Flores (2018). Constructions of copulas with given diagonal (and opposite diagonal) sections and some generalizations. Depend. Model. 6, 139-155.

[13] Fredricks, G. A. and R. B. Nelsen (2007). On the relationship between Spearman's rho and Kendall's tau for pairs of continuous random variables. J. Statist. Plann. Inference 137(7), 2143-2150.

[14] Fuchs, S. (2014). Multivariate copulas: Transformations, symmetry, order and measures of concordance. Kybernetika 50(5), 725-743.

[15] Fuchs, S. (2016). A biconvex form for copulas. Depend. Model. 4, 63-75.

[16] Fuchs, S. and K. D. Schmidt (2014). Bivariate copulas: Transformations, asymmetry and measures of concordance. Kybernetika 50(1), 109-125.

[17] Gijbels, I., V. Kika, and M. Omelka (2021). On the specification of multivariate association measures and their behaviour with increasing dimension. J. Multivariate Anal. 182, Article ID 104704, 24 pages.

[18] Gijbels, I., M. Omelka, M. Pešta, and N. Veraverbeke (2017). Score tests for covariate effects in conditional copulas. J. Multivariate Anal. 159, 111-133.

[19] Gijbels, I., M. Omelka, and N. Veraverbeke (2015). Partial and average copulas and association measures. Electron. J. Stat. 9(2), 2420-2474.

[20] Gijbels, I., M. Omelka, and N. Veraverbeke (2017). Nonparametric testing for no covariate effects in conditional copulas. Statistics 51(3), 475-509.

[21] Gijbels, I., N. Veraverbeke, and M. Omelka (2011). Conditional copulas, association measures and their applications. Comput. Statist. Data Anal. 55(5), 1919-1932.

[22] Hobæk Haff, I., K. Aas, and A. Frigessi (2010). On the simplified pair-copula construction - Simply useful or too simplistic? J. Multivariate Anal. 101(5), 1296-1310. 
[23] Hofert, M., I. Kojadinovic, M. Mächler, and J. Yan (2018). Elements of Copula Modeling with R. Springer, Cham.

[24] Joe, H. (1990). Multivariate concordance. J. Multivariate Anal. 35(1), 12-30.

[25] Mesfioui, M. and J.-F. Quessy (2008). Dependence structure of conditional Archimedean copulas. J. Multivariate Anal. 99(3), 372-385.

[26] Milgrom, P. and J. Roberts (1990). Rationalizability, learning, and equilibrium in games with strategic complementarities. Econometrica 58(6), 1255-1277.

[27] Mroz, T., S. Fuchs, and W. Trutschnig (2021). How simplifying and flexible is the simplifying assumption in pair-copula constructions - analytic answers in dimension three and a glimpse beyond. Electron. J. Stat. 15(1), 1951-1992.

[28] Nelsen, R. B. (2006). An Introduction to Copulas. Second edition. Springer, New York.

[29] Patton, A. J. (2006). Modeling asymmetric exchange rate dependence. Internat. Econom. Rev. 47(2), 527-556.

[30] Rodríguez-Lallena, J. A. and M. Úbeda-Flores (2004). A new class of bivariate copulas. Statist. Probab. Lett. 66(3), $315-325$.

[31] Sklar, A. (1959). Fonctions de répartition à $n$ dimensions et leurs marges. Publ. Inst. Statist. Univ. Paris 8, $229-231$.

[32] Spanhel, F. and M. S. Kurz (2016). The partial copula: Properties and associated dependence measures. Statist. Probab. Lett. 119, 76-83.

[33] Stöber, J., H. Joe, and C. Czado (2013). Simplified pair copula constructions - Limitations and extensions. J. Multivariate Anal. 119, 101-118.

[34] Topkis, D. M. (1978). Minimizing a submodular function on a lattice. Oper. Res. 26(2), 305-321.

[35] Veraverbeke, N., M. Omelka, and I. Gijbels (2011). Estimation of a conditional copula and association measures. Scand. J. Stat. 38(4), 766-780.

[36] Wolff, E. F. (1980). $N$-dimensional measures of dependence. Stochastica 4(3), 175-188.

[37] Wysocki, W. (2015). Kendall's tau and Spearman's rho for $n$-dimensional Archimedean copulas and their asymptotic properties. J. Nonparametr. Stat. 27(4), 442-459. 